Article

\title{
Detection of 191 Taxifolin Metabolites and Their Distribution in Rats Using HPLC-ESI-IT-TOF-MS ${ }^{\mathbf{n}}$
}

\author{
Ping Yang, Feng Xu *, Hong-Fu Li, Yi Wang, Feng-Chun Li, Ming-Ying Shang, Guang-Xue Liu, \\ Xuan Wang and Shao-Qing Cai *
}

State Key Laboratory of Natural and Biomimetic Drugs, School of Pharmaceutical Sciences, Peking University Health Science Center, Beijing 100191, China; y.p.nn@163.com (P.Y.); lihongfu6688@163.com (H.-F.L.); wangyishaanxi@163.com (Y.W.); lcxbjmu@163.com (F.-C.L.); myshang@bjmu.edu.cn (M.-Y.S.); guangxl@bjmu.edu.cn (G.-X.L.); xuanwang6818@bjmu.edu.cn (X.W.)

* Correspondence: xufeng_pharm@163.com (F.X.); sqcai@bjmu.edu.cn (S.-Q.C.); Tel.: +86-10-8280-2534 (F.X.); +86-10-8280-1693 (S.-Q.C.)

Academic Editor: Derek J. McPhee

Received: 5 July 2016; Accepted: 6 September 2016; Published: 13 September 2016

\begin{abstract}
Taxifolin is a ubiquitous bioactive constituent of foods and herbs. To thoroughly explore its metabolism in vivo, an HPLC-ESI-IT-TOF-MS ${ }^{n}$ method combined with specific metabolite detection strategy was used to detect and identify the metabolites of taxifolin in rats. Of the 191 metabolites tentatively identified, 154 were new metabolites, 69 were new compounds and 32 were dimers. This is the first report of the in vivo biotransformation of a single compound into more than 100 metabolites. Furthermore, acetylamination and pyroglutamic acid conjugation were identified as new metabolic reactions. Seventeen metabolites were found to have various taxifolin-related bioactivities. The potential targets of taxifolin and 63 metabolites were predicted using PharmMapper, with results showing that more than 60 metabolites have the same five targets. Metabolites with the same fragment pattern may have the same pharmacophore. Thus these metabolites may exert the same pharmacological effects as taxifolin through an additive effect on the same drug targets. This observation indicates that taxifolin is bioactive not only in the parent form, but also through its metabolites. These findings enhance understanding of the metabolism and effective forms of taxifolin and may provide further insight of the beneficial effects of taxifolin and its derivatives.
\end{abstract}

Keywords: taxifolin; metabolites; HPLC-ESI-IT-TOF-MS ${ }^{n}$; in vivo; additive effect at the same target

\section{Introduction}

Taxifolin (dihydroquercetin) is a bioactive flavanonol commonly found in grapes [1], citrus fruits [2], onions [2,3], green tea [1], olive oil [2], wine [1], and many other foods [2], as well as several herbs (such as milk thistle [4], French maritime bark [5], Douglas fir bark [6], and Smilacis Glabrae Rhizoma [7]). It is also widely used as a food additive and can be found in health supplement products such as silymarin (Legalon ${ }^{\mathrm{TM}}$ ), Pycnogenol ${ }^{\circledR}$ and Venoruton ${ }^{\circledR}$ [8].

As a ubiquitous constituent of foods and herbs, taxifolin is consumed regularly in the human diet and exerts a wide range of biochemical and pharmacological effects; these include antioxidant $[9,10]$, antitumor [11] and anti-inflammatory effects [12], the prevention of Alzheimer's disease [13], antidiabetic [14,15], antiviral [16], antimicrobial [17], hepatoprotective [18], cardioprotective [15,19], neuroprotective [20] and immunoregulatory effects [21], and xanthine oxidase inhibition [22]. Additionally, experimental data indicate that taxifolin use is safe and nontoxic [2,23].

It has been reported that the effective forms of flavonoids are not necessarily the natural phytochemical forms, but the metabolites [24-26] arising from them in vivo. It is well established that 
conjugation reactions with glucuronic acid, sulphuric acid, and their mixtures are the most common type of metabolic pathways for flavonoids $[27,28]$. Some studies have shown that phase II metabolites possess certain pharmacological activities such as anti-inflammatory, antioxidant and antitumor effects, and can interact with metabolic enzymes and transporters [26-28].

Like other flavonoids, taxifolin can be metabolized, absorbed, and circulated in conjugate form throughout the body, thus exerting beneficial effects in target tissues [29-31]. According to our previous studies, a single bioactive constituent of herbs can produce more than 50 [32] or 80 [33] metabolites in vivo. However, until now, only about 27 in vitro and in vivo metabolites of taxifolin have been described. The predominant metabolites include 3,4-dihydroxyphenylacetic acid [23,34], phloroglucinol [34], m-hydroxyphenylacetic acid [23], 3-methoxy-4-hydroxylphenylacetic acid [23], a dehydroxylation metabolite [35], methylation product [30,35], sulphate [35], glucuronide [35], methylated glucuronides [35], a diastereomer [30], methylation isomer [30] and dehydration metabolites [30]. Accordingly, the biotransformation of taxifolin and the biological activities of its metabolites still need further investigation.

The apparent permeability of taxifolin across Caco- 2 cell monolayers (a widely used in vitro model of the human small intestinal mucosa) was shown to be less than $1 \times 10^{-6} \mathrm{~cm} / \mathrm{s}$ [36], and the absolute bioavailability of taxifolin was reported as $0.17 \%$ in rats [37]. The bioavailability of taxifolin was $36 \%$ in rabbits upon detection of total conjugated and free taxifolin in plasma following enzymatic hydrolysis [38]. The question therefore remains as to how taxifolin exerts its biochemical and pharmacological effects with such low bioavailability. Previous findings indicate that the parent compound of taxifolin is found at low levels in the blood, and that conjugates represent the main forms in vivo. Moreover, the urinary excretion of taxifolin was found to be only $0.24 \%$ of the dosage [30]. Therefore, we believe that taxifolin may be easily metabolized and that its metabolites are the prevalent form in vivo, although limited information is available on metabolism of taxifolin in vivo. To gain a more comprehensive understanding of taxifolin metabolism and its effective forms [39], mechanisms of action, and pharmacological effects in vivo, it is necessary to thoroughly profile its metabolites and determine their distribution. Accordingly, we used high-performance liquid chromatography with electrospray ionization ion trap time-of-flight multistage mass spectrometry (HPLC-ESI-IT-TOF-MS ${ }^{n}$ ) combined with a specific metabolite analysis strategy to profile and identify the metabolites of taxifolin and study their distribution in rats.

\section{Results and Discussion}

\subsection{Identification of Taxifolin in Rats and Study on the Fragmentation Behaviours of Taxifolin and Reference Compounds}

Taxifolin ([M - H $]^{-}$at $m / z$ 303.0510, molecular formula $\left.\mathrm{C}_{15} \mathrm{H}_{12} \mathrm{O}_{7}\right)$ was unambiguously identified in rat plasma, urine, faeces and eight organ samples by comparing its retention time $\left(t_{R}=41.023 \mathrm{~min}\right)$ and $\mathrm{MS}^{\mathrm{n}}$ data with the reference compound. To facilitate the identification of metabolites, the fragmentation characteristics of taxifolin in the negative ion mode (NI) were observed and analysed (Supplemental Table S1 and Figures S1 and S2). The characteristic fragment ions of taxifolin in NI were $m / z 285.0407\left(\left[\mathrm{M}-\mathrm{H}-\mathrm{H}_{2} \mathrm{O}\right]^{-}\right), m / z 241.0524\left(\left[\mathrm{M}-\mathrm{H}-\mathrm{H}_{2} \mathrm{O}-\mathrm{CO}_{2}\right]^{-}\right), m / z 177.0253$ $\left({ }^{1,4} \mathrm{~B}^{-}-2 \mathrm{H}\right), m / z 175.0424\left(\left[\mathrm{M}-\mathrm{H}-\mathrm{H}_{2} \mathrm{O}-\mathrm{C}_{3} \mathrm{O}_{2}-\mathrm{C}_{2} \mathrm{H}_{2} \mathrm{O}\right]^{-}\right)$and $m / z 125.0290\left({ }^{1,4} \mathrm{~A}^{-}+2 \mathrm{H}\right)$ in its $\mathrm{MS}^{2}$ spectrum. Quercetin $\left(\mathrm{C}_{15} \mathrm{H}_{10} \mathrm{O}_{7}\right)$ showed characteristic fragment ions at $\mathrm{m} / \mathrm{z} 229.0526, \mathrm{~m} / \mathrm{z} 211.0386$, $m / z 179.0015, m / z 151.0061$ and $m / z 107.0230$ in its $\mathrm{MS}^{2}$ spectrum. Dihydrokaempferol $\left(\mathrm{C}_{15} \mathrm{H}_{12} \mathrm{O}_{6}\right)$ showed characteristic fragment ions at $\mathrm{m} / \mathrm{z} 269.0431, \mathrm{~m} / \mathrm{z} 259.0613, \mathrm{~m} / \mathrm{z} 243.0663, \mathrm{~m} / \mathrm{z} 201.0564$, $m / z 173.0622$ and $m / z 125.0275$ in its MS$^{2}$ spectrum.

\subsection{Identification of 191 Metabolites of Taxifolin in Rats}

Metabolites of taxifolin in rats were identified on the basis of knowledge of taxifolin metabolism and the strategy proposed in our previous study [39]. The metabolic reactions were identified according 
to characteristic neutral losses. Compared with the parent compound, the formation of metabolites with mass shifts of $+15.99 \mathrm{Da}(\mathrm{O}),-15.99 \mathrm{Da}(\mathrm{O}),+14.01 \mathrm{Da}\left(\mathrm{CH}_{2}\right),-2.01 \mathrm{Da}\left(\mathrm{H}_{2}\right),+2.01 \mathrm{Da}\left(\mathrm{H}_{2}\right)$, $-18.01 \mathrm{Da}\left(\mathrm{H}_{2} \mathrm{O}\right),+18.01 \mathrm{Da}\left(\mathrm{H}_{2} \mathrm{O}\right),+79.95 \mathrm{Da}\left(\mathrm{SO}_{3}\right)$ and $+176.03 \mathrm{Da}\left(\mathrm{C}_{6} \mathrm{H}_{8} \mathrm{O}_{6}\right)$ indicated hydroxylation, dehydroxylation, methylation, dehydrogenation, hydrogenation, dehydration, hydration, sulphation, and glucuronidation, respectively. The molecular formulae were predicted based on HRMS data, and the specific type and structure of metabolites were identified by the fragmentation characteristics in their NI MS ${ }^{\mathrm{n}}$ spectra. In total, 191 metabolites (including 127 metabolites in urine, 83 metabolites in plasma, 43 metabolites in faeces and 46 metabolites in eight organs) of taxifolin were tentatively identified (Table 1) by careful MS ${ }^{n}$ data analysis, and their existence was further confirmed by comparing the corresponding extracted ion chromatograms (EICs) of drug and blank groups. The detailed LC-MS data are summarized in Table 1 and Table S1, with potential metabolic pathways of taxifolin shown in Figure 1. Metabolic reactions are summarized in Table 2. Among the 191 metabolites, 154 were new metabolites of taxifolin, and 69 metabolites were new compounds that could not be found in the SciFinder database, including 12 taxifolin conjugates, 22 methyl taxifolin derivatives, four phenolic acid derivatives, and 31 dimers. The 191 metabolites were divided into eight categories: 32 metabolites having the aglycone of taxifolin or its isomers, 37 metabolites having the aglycone of methyl taxifolin, 34 metabolites having the aglycone of quercetin, nine metabolites having the aglycone of dehydroxylated taxifolin, four metabolites formed through dehydration and glucuronidation, five metabolites having the aglycone of hydrogenated taxifolin, 38 metabolites having the aglycone of phenolic acid derivatives and 32 metabolites formed through dimerization.

\subsubsection{Identification of 32 Metabolites (M1-M32) Having the Aglycone of Taxifolin or Its Isomers}

In total, 32 metabolites (12 new compounds) having the aglycone of taxifolin or its isomers were identified in the drug group, including two isomers and 30 conjugates of taxifolin or its isomers. Isomerization metabolites M1 and M2 had the same molecular formula and similar fragmentation behaviour as taxifolin. Because C-2 and C-3 are chiral centres, taxifolin has four stereoisomers [7], therefore, these metabolites were tentatively identified as stereoisomers of taxifolin. As for the taxifolin conjugates M3-M32, in the NI MS ${ }^{2}$ spectra of M3-M32, the same [aglycone $\left.-\mathrm{H}\right]^{-}(\mathrm{m} / \mathrm{z} 303.05)$ was observed, with identical molecular formula and fragmentation behaviour to taxifolin. We therefore deduced that the metabolites were conjugates of taxifolin. According to their characteristic neutral losses, M3-M11 were identified as sulphates of taxifolin or its isomers. Conjugates M12-M15 were identified as taxifolin disulphates. The molecular formula of $\mathbf{M 1 6}$ was determined to be $\mathrm{C}_{20} \mathrm{H}_{19} \mathrm{NO}_{13} \mathrm{~S}$ from its HRMS data. The base peak ion at $m / z 383.0083$ was formed by neutral loss of $129.05 \mathrm{Da}$ $\left(\mathrm{C}_{5} \mathrm{H}_{7} \mathrm{NO}_{3}\right)$. According to our previous research [40], we deduced that the metabolic reaction was amino acid conjugation and the lost fragment $\mathrm{C}_{5} \mathrm{H}_{7} \mathrm{NO}_{3}$ was speculated to be pyroglutamic acid (the most referenced compound having the molecular formula of $\mathrm{C}_{5} \mathrm{H}_{7} \mathrm{NO}_{3}$ ) based on the SciFinder academic database. Hence, M16 was tentatively identified as taxifolin sulphate and pyroglutamic acid conjugate. M17-M25 were identified as glucuronides of taxifolin or its isomers, and M26-M32 were identified as taxifolin glucuronide sulphates.

\subsubsection{Identification of 37 Metabolites (M33-M69) Having the Aglycone of Methyl Taxifolin}

In total, 37 metabolites (22 new compounds) having the aglycone of methyl taxifolin or its isomer were found in the drug group, including four methyltaxifolin isomers, 23 conjugates of methyl taxifolin or its isomers, four conjugates of methyl and hydroxylated taxifolin, and six conjugates of methyl and dihydroxylated taxifolin.

The molecular formulae of M33-M36 were calculated to be $\mathrm{C}_{16} \mathrm{H}_{14} \mathrm{O}_{7}$, which is $14.01 \mathrm{Da}\left(\mathrm{CH}_{2}\right)$ more than that of taxifolin. Hence, these compounds were identified as methylated taxifolin. Generally, the hydroxyl group at the C-5 position is not readily metabolized [40]. Therefore, the sites of methylation were found to be the hydroxyl groups of the C-3, $7,3^{\prime}$ and $4^{\prime}$ positions of taxifolin. 
Table 1. Retention time $\left(t_{R}\right)$, HRMS data, molecular formula, and identification of taxifolin and its 191 metabolites in rats urine, plasma, faeces by HPLC-ESI-IT-TOF-MS ${ }^{n}$.

\begin{tabular}{|c|c|c|c|c|c|c|c|c|c|c|c|c|}
\hline No. & $t_{R}(\min )$ & Formula & Ion & Meas. $m / z$ & Pred. $m / z$ & Diff (ppm) & DBE & Urine & $\begin{array}{l}\text { Plasma } \\
\text { Plo }\end{array}$ & Faeces & Identification Level & Identification \\
\hline TAX & 41.023 & $\mathrm{C}_{15} \mathrm{H}_{12} \mathrm{O}_{7}$ & {$[\mathrm{M}-\mathrm{H}]^{-}$} & 303.0521 & 303.0510 & 3.63 & 10 & $\Delta$ & $\Delta$ & $\Delta$ & Level 1 & Taxifolin (parent compound) \\
\hline & & & & & \multicolumn{8}{|c|}{ Metabolites having the aglycone of taxifolin or its isomers (M1-M32); two bioactive metabolites } \\
\hline$M 1^{a, b}$ & 40.508 & $\mathrm{C}_{15} \mathrm{H}_{12} \mathrm{O}_{7}$ & {$[\mathrm{M}-\mathrm{H}]^{-}$} & 303.0502 & 303.0510 & -2.64 & 10 & - & $\Delta$ & $\Delta$ & Level 2 & Taxifolin isomer 1 \\
\hline M2 a,b & 42.883 & $\mathrm{C}_{15} \mathrm{H}_{12} \mathrm{O}_{7}$ & {$[\mathrm{M}-\mathrm{H}]^{-}$} & 303.0517 & 303.0510 & 2.31 & 10 & $\Delta$ & $\Delta$ & $\Delta$ & Level 2 & Taxifolin isomer 2 \\
\hline $\mathbf{M}^{\mathrm{b}}$ & 21.517 & $\mathrm{C}_{15} \mathrm{H}_{12} \mathrm{O}_{10} \mathrm{~S}$ & {$[\mathrm{M}-\mathrm{H}]^{-}$} & 383.0080 & 383.0078 & 0.52 & 10 & $\Delta$ & - & - & Level 2 & Taxifolin sulphate 1 \\
\hline M4 ${ }^{\mathrm{b}}$ & 31.242 & $\mathrm{C}_{15} \mathrm{H}_{12} \mathrm{O}_{10} \mathrm{~S}$ & {$[\mathrm{M}-\mathrm{H}]^{-}$} & 383.0089 & 383.0078 & 2.87 & 10 & $\Delta$ & - & - & Level 2 & Taxifolin sulphate 2 \\
\hline M5 ${ }^{b}$ & 32.145 & $\mathrm{C}_{15} \mathrm{H}_{12} \mathrm{O}_{10} \mathrm{~S}$ & {$[\mathrm{M}-\mathrm{H}]^{-}$} & 383.0073 & 383.0078 & -1.31 & 10 & & $\Delta$ & $\Delta$ & Level 2 & Taxifolin sulphate 3 \\
\hline$M 6^{b}$ & 35.292 & $\mathrm{C}_{15} \mathrm{H}_{12} \mathrm{O}_{10} \mathrm{~S}$ & {$[\mathrm{M}-\mathrm{H}]^{-}$} & 383.0078 & 383.0078 & 0.00 & 10 & $\Delta$ & & - & Level 2 & Taxifolin sulphate 4 \\
\hline$M 7^{b}$ & 36.717 & $\mathrm{C}_{15} \mathrm{H}_{12} \mathrm{O}_{10} \mathrm{~S}$ & {$[\mathrm{M}-\mathrm{H}]^{-}$} & 383.0079 & 383.0078 & 0.26 & 10 & $\Delta$ & $\Delta$ & $\Delta$ & Level 2 & Taxifolin sulphate 5 \\
\hline M8 ${ }^{\mathrm{b}}$ & 37.925 & $\mathrm{C}_{15} \mathrm{H}_{12} \mathrm{O}_{10} \mathrm{~S}$ & {$[\mathrm{M}-\mathrm{H}]^{-}$} & 383.0070 & 383.0078 & -2.09 & 10 & $\Delta$ & $\Delta$ & & Level 2 & Taxifolin sulphate 6 \\
\hline M9 ${ }^{\mathrm{b}}$ & 39.375 & $\mathrm{C}_{15} \mathrm{H}_{12} \mathrm{O}_{10} \mathrm{~S}$ & {$[\mathrm{M}-\mathrm{H}]^{-}$} & 383.0087 & 383.0078 & 2.35 & 10 & $\Delta$ & & $\Delta$ & Level 2 & Taxifolin sulphate 7 \\
\hline M10 ${ }^{b}$ & 41.192 & $\mathrm{C}_{15} \mathrm{H}_{12} \mathrm{O}_{10} \mathrm{~S}$ & {$[\mathrm{M}-\mathrm{H}]^{-}$} & 383.0086 & 383.0078 & 2.09 & 10 & $\Delta$ & $\Delta$ & $\Delta$ & Level 2 & Taxifolin sulphate 8 \\
\hline M11 ${ }^{\mathrm{b}}$ & 43.000 & $\mathrm{C}_{15} \mathrm{H}_{12} \mathrm{O}_{10} \mathrm{~S}$ & {$[\mathrm{M}-\mathrm{H}]^{-}$} & 383.0082 & 383.0078 & 1.04 & 10 & $\Delta$ & $\Delta$ & $\Delta$ & Level 2 & Taxifolin sulphate 9 \\
\hline M12 ${ }^{\mathrm{c}}$ & 24.592 & $\mathrm{C}_{15} \mathrm{H}_{12} \mathrm{O}_{13} \mathrm{~S}_{2}$ & {$[\mathrm{M}-\mathrm{H}]^{-}$} & 462.9644 & 462.9647 & -0.65 & 10 & $\Delta$ & & 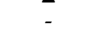 & Level 3 & Taxifolin disulphate 1 \\
\hline$M 13^{\mathrm{C}}$ & 27.458 & $\mathrm{C}_{15} \mathrm{H}_{12} \mathrm{O}_{13} \mathrm{~S}_{2}$ & {$[\mathrm{M}-\mathrm{H}]^{-}$} & 462.9670 & 462.9647 & 4.97 & 10 & $\Delta$ & - & - & Level 3 & $\begin{array}{l}\text { Taxifolin disulphate } 2 \\
\text { S }\end{array}$ \\
\hline M14 ${ }^{\mathrm{C}}$ & 31.075 & $\mathrm{C}_{15} \mathrm{H}_{12} \mathrm{O}_{13} \mathrm{~S}_{2}$ & {$[\mathrm{M}-\mathrm{H}]^{-}$} & 462.9639 & 462.9647 & -1.73 & 10 & $\Delta$ & - & - & Level 3 & Taxifolin disulphate 3 \\
\hline M15 ${ }^{\mathrm{c}}$ & 39.767 & $\mathrm{C}_{15} \mathrm{H}_{12} \mathrm{O}_{13} \mathrm{~S}_{2}$ & {$[\mathrm{M}-\mathrm{H}]^{-}$} & 462.9656 & 462.9647 & 1.94 & 10 & $\Delta$ & - & - & Level 3 & $\begin{array}{l}\text { Taxifolin disulphate } 4 \\
\end{array}$ \\
\hline M16 ${ }^{\mathrm{c}}$ & 16.252 & $\mathrm{C}_{20} \mathrm{H}_{19} \mathrm{NO}_{13} \mathrm{~S}$ & {$[\mathrm{M}-\mathrm{H}]^{-}$} & 512.0509 & 512.0504 & 0.98 & 12 & $\Delta$ & - & - & Level 3 & Taxifolin sulphate and pyroglutamic acid conjugate \\
\hline M17 ${ }^{b}$ & 15.408 & $\mathrm{C}_{21} \mathrm{H}_{20} \mathrm{O}_{13}$ & {$[\mathrm{M}-\mathrm{H}]^{-}$} & 479.0834 & 479.0831 & 0.63 & 12 & $\Delta$ & - & - & Level 2 & $\begin{array}{l}\text { Taxifolin glucuronide } 1\end{array}$ \\
\hline M18 & 18.637 & $\mathrm{C}_{21} \mathrm{H}_{20} \mathrm{O}_{13}$ & {$[\mathrm{M}-\mathrm{H}]^{-}$} & 479.0850 & 479.0831 & 3.97 & 12 & - & $\Delta$ & - & $\begin{array}{l}\text { Level } 2 \\
\text { Level }\end{array}$ & Taxifolin glucuronide 2 \\
\hline M19 & 20.253 & $\mathrm{C}_{21} \mathrm{H}_{20} \mathrm{O}_{13}$ & {$[\mathrm{M}-\mathrm{H}]^{-}$} & 479.0847 & 479.0831 & 3.34 & 12 & $\Delta$ & $\Delta$ & - & Level 2 & Taxifolin glucuronide 3 \\
\hline $\mathrm{M} 20^{\mathrm{b}}$ & 21.370 & $\mathrm{C}_{21} \mathrm{H}_{20} \mathrm{O}_{13}$ & {$[\mathrm{M}-\mathrm{H}]^{-}$} & 479.0843 & 479.0831 & 2.50 & 12 & $\Delta$ & $\Delta$ & - & Level 2 & Taxifolin glucuronide 4 \\
\hline M21 ${ }^{b}$ & 22.267 & $\mathrm{C}_{21} \mathrm{H}_{20} \mathrm{O}_{13}$ & {$[\mathrm{M}-\mathrm{H}]^{-}$} & 479.0838 & 479.0831 & 1.46 & 12 & $\Delta$ & $\Delta$ & - & Level 2 & Taxifolin glucuronide 5 \\
\hline M22 ${ }^{b}$ & 22.587 & $\mathrm{C}_{21} \mathrm{H}_{20} \mathrm{O}_{13}$ & {$[\mathrm{M}-\mathrm{H}]^{-}$} & 479.0847 & 479.0831 & 3.34 & 12 & & $\Delta$ & - & Level 2 & Taxifolin glucuronide 6 \\
\hline $\mathrm{M} 23^{\mathrm{b}}$ & 31.862 & $\mathrm{C}_{21} \mathrm{H}_{20} \mathrm{O}_{13}$ & {$[\mathrm{M}-\mathrm{H}]^{-}$} & 479.0830 & 479.0831 & $\begin{array}{c}5.04 \\
-0.21\end{array}$ & 12 & $\Delta$ & $\Delta$ & - & Level 2 & Taxifolin glucuronide 7 \\
\hline M24 ${ }^{\text {b }}$ & 34.742 & $\mathrm{C}_{21} \mathrm{H}_{20} \mathrm{O}_{13}$ & {$[\mathrm{M}-\mathrm{H}]^{-}$} & 479.0832 & 479.0831 & 0.21 & 12 & $\Delta$ & - & - & Level 2 & Taxifolin glucuronide 8 \\
\hline M25 ${ }^{b}$ & 37.267 & $\mathrm{C}_{21} \mathrm{H}_{20} \mathrm{O}_{13}$ & {$[\mathrm{M}-\mathrm{H}]^{-}$} & $\begin{array}{l}479.0032 \\
479.0834\end{array}$ & 479.0831 & $\begin{array}{l}0.21 \\
0.63\end{array}$ & 12 & $\Delta$ & $\bar{\Delta}$ & - & $\begin{array}{l}\text { Level } 2 \\
\text { Level }\end{array}$ & $\begin{array}{l}\text { Taxifolin glucuronide } 9 \\
\text { Taxide }\end{array}$ \\
\hline $\mathrm{M} 26^{\mathrm{c}}$ & 13.888 & $\mathrm{C}_{21} \mathrm{H}_{20} \mathrm{O}_{16} \mathrm{~S}$ & {$[\mathrm{M}-\mathrm{H}]^{-}$} & 559.0388 & 559.0399 & $\begin{array}{l}0.03 \\
-1.97\end{array}$ & 12 & $=$ & $\Delta$ & - & $\begin{array}{l}\text { Level } 3 \\
\text { Lever }\end{array}$ & Taxifolin glucuronide sulphate 1 \\
\hline M27 ${ }^{\mathrm{c}}$ & 16.703 & $\mathrm{C}_{21} \mathrm{H}_{20} \mathrm{O}_{16} \mathrm{~S}$ & {$[\mathrm{M}-\mathrm{H}]^{-}$} & 559.0423 & 559.0399 & 4.29 & 12 & $\Delta$ & $\Delta$ & - & Level 3 & Taxifolin glucuronide sulphate 2 \\
\hline$M 28^{\mathrm{C}}$ & 19.928 & $\mathrm{C}_{21} \mathrm{H}_{20} \mathrm{O}_{16} \mathrm{~S}$ & {$[\mathrm{M}-\mathrm{H}]^{-}$} & 559.0406 & 559.0399 & 1.25 & 12 & $\Delta$ & $\Delta$ & - & Level 3 & Taxifolin glucuronide sulphate 3 \\
\hline M29 $9^{\mathrm{C}}$ & 21.812 & $\mathrm{C}_{21} \mathrm{H}_{20} \mathrm{O}_{16} \mathrm{~S}$ & {$[\mathrm{M}-\mathrm{H}]^{-}$} & 559.0411 & 559.0399 & 2.15 & 12 & & $\Delta$ & - & Level 3 & Taxifolin glucuronide sulphate 4 \\
\hline $\mathrm{M} 30^{\mathrm{C}}$ & 23.087 & $\mathrm{C}_{21} \mathrm{H}_{20} \mathrm{O}_{16} \mathrm{~S}$ & {$[\mathrm{M}-\mathrm{H}]^{-}$} & 559.0418 & 559.0399 & 3.40 & 12 & $\Delta$ & $\Delta$ & - & Level 3 & Taxifolin glucuronide sulphate 5 \\
\hline M31 ${ }^{\mathrm{c}}$ & 24.762 & $\mathrm{C}_{21} \mathrm{H}_{20} \mathrm{O}_{16} \mathrm{~S}$ & {$[\mathrm{M}-\mathrm{H}]^{-}$} & 559.0425 & 559.0399 & 4.65 & 12 & & $\Delta$ & - & Level 3 & Taxifolin glucuronide sulphate 6 \\
\hline M32 ${ }^{\mathrm{C}}$ & 25.797 & $\mathrm{C}_{21} \mathrm{H}_{20} \mathrm{O}_{16} \mathrm{~S}$ & {$[\mathrm{M}-\mathrm{H}]^{-}$} & 559.0411 & 559.0399 & 2.86 & 12 & - & & - & Level 3 & Taxifolin glucuronide sulphate 7 \\
\hline
\end{tabular}


Table 1. Cont.

\begin{tabular}{|c|c|c|c|c|c|c|c|c|c|c|c|c|}
\hline No. & $t_{R}(\min )$ & Formula & Ion & Meas. $m / z$ & Pred. $m / z$ & Diff (ppm) & DBE & Urine & Plasma & Faeces & Identification Level & Identification \\
\hline & & & & & \multicolumn{8}{|c|}{ Metabolites having the aglycone of methyl taxifolin (M33-M69) } \\
\hline$M 33^{b, d}$ & 50.292 & $\mathrm{C}_{16} \mathrm{H}_{14} \mathrm{O}_{7}$ & {$[\mathrm{M}-\mathrm{H}]^{-}$} & 317.0675 & 317.0667 & 2.52 & 10 & $\Delta$ & $\Delta$ & $\Delta$ & Level 2 & 3'-O-Methyltaxifolin \\
\hline M34 b,d & 51.350 & $\mathrm{C}_{16} \mathrm{H}_{14} \mathrm{O}_{7}$ & {$[\mathrm{M}-\mathrm{H}]^{-}$} & 317.0673 & 317.0667 & 1.89 & 10 & $\Delta$ & $\Delta$ & $\Delta$ & Level 2 & $4^{\prime}-O-$ Methyltaxifolin \\
\hline $\mathrm{M} 35^{\mathrm{b}, \mathrm{d}}$ & 52.875 & $\mathrm{C}_{16} \mathrm{H}_{14} \mathrm{O}_{7}$ & {$[\mathrm{M}-\mathrm{H}]^{-}$} & 317.0667 & 317.0667 & 0.00 & 10 & $\Delta$ & $\Delta$ & $\Delta$ & Level 2 & 7-O-Methyltaxifolin \\
\hline $\mathrm{M} 36^{\mathrm{b}, \mathrm{d}}$ & 53.592 & $\mathrm{C}_{16} \mathrm{H}_{14} \mathrm{O}_{7}$ & {$[\mathrm{M}-\mathrm{H}]^{-}$} & 317.0660 & 317.0667 & -2.21 & 10 & $\Delta$ & - & - & Level 2 & 3-O-Methyltaxifolin \\
\hline M37 ${ }^{\mathrm{c}}$ & 28.575 & $\mathrm{C}_{16} \mathrm{H}_{14} \mathrm{O}_{10} \mathrm{~S}$ & {$[\mathrm{M}-\mathrm{H}]^{-}$} & 397.0243 & 397.0235 & 2.01 & 10 & $\Delta$ & - & - & Level 3 & Methyl taxifolin sulphate 1 \\
\hline M38 ${ }^{\mathrm{C}}$ & 33.942 & $\mathrm{C}_{16} \mathrm{H}_{14} \mathrm{O}_{10} \mathrm{~S}$ & {$[\mathrm{M}-\mathrm{H}]^{-}$} & 397.0240 & 397.0235 & 1.26 & 10 & $\Delta$ & $\Delta$ & - & Level 3 & Methyl taxifolin sulphate 2 \\
\hline M39 ${ }^{\mathrm{c}}$ & 34.420 & $\mathrm{C}_{16} \mathrm{H}_{14} \mathrm{O}_{10} \mathrm{~S}$ & {$[\mathrm{M}-\mathrm{H}]^{-}$} & 397.0247 & 397.0235 & 3.02 & 10 & - & $\Delta$ & - & Level 3 & Methyl taxifolin sulphate 3 \\
\hline $\mathrm{M} 40^{\mathrm{c}}$ & 35.858 & $\mathrm{C}_{16} \mathrm{H}_{14} \mathrm{O}_{10} \mathrm{~S}$ & {$[\mathrm{M}-\mathrm{H}]^{-}$} & 397.0253 & 397.0235 & 4.53 & 10 & $\Delta$ & - & - & Level 3 & Methyl taxifolin sulphate 4 \\
\hline $\mathrm{M}_{4}{ }^{\mathrm{c}}$ & 38.092 & $\mathrm{C}_{16} \mathrm{H}_{14} \mathrm{O}_{10} \mathrm{~S}$ & {$[\mathrm{M}-\mathrm{H}]^{-}$} & 397.0241 & 397.0235 & 1.51 & 10 & $\Delta$ & - & - & Level 3 & Methyl taxifolin sulphate 5 \\
\hline $\mathrm{M} 42^{\mathrm{c}}$ & 40.283 & $\mathrm{C}_{16} \mathrm{H}_{14} \mathrm{O}_{10} \mathrm{~S}$ & {$[\mathrm{M}-\mathrm{H}]^{-}$} & 397.0233 & 397.0235 & -0.50 & 10 & $\Delta$ & $\Delta$ & $\Delta$ & Level 3 & Methyl taxifolin sulphate 6 \\
\hline $\mathrm{M} 43^{\mathrm{c}}$ & 41.817 & $\mathrm{C}_{16} \mathrm{H}_{14} \mathrm{O}_{10} \mathrm{~S}$ & {$[\mathrm{M}-\mathrm{H}]^{-}$} & 397.0241 & 397.0235 & 1.51 & 10 & $\Delta$ & $\Delta$ & $\Delta$ & Level 3 & Methyl taxifolin sulphate 7 \\
\hline $\mathrm{M} 44^{\mathrm{c}}$ & 42.717 & $\mathrm{C}_{16} \mathrm{H}_{14} \mathrm{O}_{10} \mathrm{~S}$ & {$[\mathrm{M}-\mathrm{H}]^{-}$} & 397.0230 & 397.0235 & -1.26 & 10 & $\bar{\Delta}$ & & $=$ & Level 3 & Methyl taxifolin sulphate 8 \\
\hline$M 45^{c}$ & 43.600 & $\mathrm{C}_{16} \mathrm{H}_{14} \mathrm{O}_{10} \mathrm{~S}$ & {$[\mathrm{M}-\mathrm{H}]^{-}$} & 397.0235 & 397.0235 & 0.00 & 10 & $\bar{\Delta}$ & $\Delta$ & - & Level 3 & Methyl taxifolin sulphate 9 \\
\hline $\mathrm{M} 46^{\mathrm{C}}$ & 45.558 & $\mathrm{C}_{16} \mathrm{H}_{14} \mathrm{O}_{10} \mathrm{~S}$ & {$[\mathrm{M}-\mathrm{H}]^{-}$} & 397.0238 & 397.0235 & 0.76 & 10 & $\Delta$ & $\Delta$ & $\Delta$ & Level 3 & Methyl taxifolin sulphate 10 \\
\hline M47 b & 23.520 & $\mathrm{C}_{22} \mathrm{H}_{22} \mathrm{O}_{13}$ & {$[\mathrm{M}-\mathrm{H}]^{-}$} & 493.0973 & 493.0988 & -3.04 & 12 & $=$ & $\Delta$ & $=$ & Level 2 & Methyl taxifolin glucuronide 1 \\
\hline $\mathrm{M} 48^{\mathrm{b}}$ & 25.212 & $\mathrm{C}_{22} \mathrm{H}_{22} \mathrm{O}_{13}$ & {$[\mathrm{M}-\mathrm{H}]^{-}$} & 493.1012 & 493.0988 & 4.87 & 12 & - & $\Delta$ & - & Level 2 & Methyl taxifolin glucuronide 2 \\
\hline M49 b & 26.687 & $\mathrm{C}_{22} \mathrm{H}_{22} \mathrm{O}_{13}$ & {$[\mathrm{M}-\mathrm{H}]^{-}$} & 493.1012 & 493.0988 & 4.87 & 12 & - & $\Delta$ & - & Level 2 & Methyl taxifolin glucuronide 3 \\
\hline $\mathrm{M} 50^{\mathrm{b}}$ & 30.383 & $\mathrm{C}_{22} \mathrm{H}_{22} \mathrm{O}_{13}$ & {$[\mathrm{M}-\mathrm{H}]^{-}$} & 493.1012 & 493.0988 & 4.87 & 12 & $\Delta$ & $\Delta$ & - & Level 2 & Methyl taxifolin glucuronide 4 \\
\hline M51 b & 33.395 & $\mathrm{C}_{22} \mathrm{H}_{22} \mathrm{O}_{13}$ & {$[\mathrm{M}-\mathrm{H}]^{-}$} & 493.1007 & 493.0988 & 3.85 & 12 & - & $\Delta$ & - & Level 2 & Methyl taxifolin glucuronide 5 \\
\hline M52 ${ }^{b}$ & 35.692 & $\mathrm{C}_{22} \mathrm{H}_{22} \mathrm{O}_{13}$ & {$[\mathrm{M}-\mathrm{H}]^{-}$} & 493.0998 & 493.0988 & 2.03 & 12 & $\Delta$ & $\Delta$ & - & Level 2 & Methyl taxifolin glucuronide 6 \\
\hline $\mathrm{M}_{5} 3^{\mathrm{b}}$ & 36.025 & $\mathrm{C}_{22} \mathrm{H}_{22} \mathrm{O}_{13}$ & {$[\mathrm{M}-\mathrm{H}]^{-}$} & 493.1004 & 493.0988 & 3.24 & 12 & $\Delta$ & - & - & Level 2 & Methyl taxifolin glucuronide 7 \\
\hline M54 b & 37.600 & $\mathrm{C}_{22} \mathrm{H}_{22} \mathrm{O}_{13}$ & {$[\mathrm{M}-\mathrm{H}]^{-}$} & 493.0998 & 493.0988 & 2.03 & 12 & $\Delta$ & - & - & Level 2 & Methyl taxifolin glucuronide 8 \\
\hline $\mathrm{M} 55^{\mathrm{b}}$ & 42.375 & $\mathrm{C}_{22} \mathrm{H}_{22} \mathrm{O}_{13}$ & {$[\mathrm{M}-\mathrm{H}]^{-}$} & 493.1008 & 493.0988 & 4.06 & 12 & $\Delta$ & - & - & Level 2 & Methyl taxifolin glucuronide 9 \\
\hline M56 & 34.742 & $\mathrm{C}_{22} \mathrm{H}_{22} \mathrm{O}_{16} \mathrm{~S}$ & {$[\mathrm{M}-\mathrm{H}]^{-}$} & 573.0560 & 573.0556 & 0.70 & 12 & $\Delta$ & - & - & Level 2 & Methyl taxifolin glucuronide sulphate 1 \\
\hline M57 & 37.158 & $\mathrm{C}_{22} \mathrm{H}_{22} \mathrm{O}_{16} \mathrm{~S}$ & {$[\mathrm{M}-\mathrm{H}]^{-}$} & 573.0533 & 573.0556 & -4.01 & 12 & $\Delta$ & - & - & Level 2 & Methyl taxifolin glucuronide sulphate 2 \\
\hline M58 ${ }^{\mathrm{c}}$ & 16.490 & $\mathrm{C}_{21} \mathrm{H}_{21} \mathrm{NO}_{10}$ & {$[\mathrm{M}-\mathrm{H}]^{-}$} & 446.1107 & 446.1093 & 3.14 & 12 & $\Delta$ & - & - & Level 3 & Methyl taxifolin pyroglutamic acid conjugate 1 \\
\hline $\mathrm{M}_{59^{\mathrm{c}}}$ & 18.483 & $\mathrm{C}_{21} \mathrm{H}_{21} \mathrm{NO}_{10}$ & {$[\mathrm{M}-\mathrm{H}]^{-}$} & 446.1086 & 446.1093 & -1.57 & 12 & $\Delta$ & - & - & Level 3 & Methyl taxifolin pyroglutamic acid conjugate 2 \\
\hline $\mathrm{M} 60^{\mathrm{c}}$ & 37.848 & $\mathrm{C}_{16} \mathrm{H}_{14} \mathrm{O}_{11} \mathrm{~S}$ & {$[\mathrm{M}-\mathrm{H}]^{-}$} & 413.0200 & 413.0184 & 3.87 & 10 & & $\Delta$ & - & Level 3 & Hydroxylated methyl taxifolin sulphate 1 \\
\hline M61 ${ }^{\mathrm{c}}$ & 41.943 & $\mathrm{C}_{16} \mathrm{H}_{14} \mathrm{O}_{11} \mathrm{~S}$ & {$[\mathrm{M}-\mathrm{H}]^{-}$} & 413.0175 & 413.0184 & -2.18 & 10 & - & $\Delta$ & - & Level 3 & Hydroxylated methyl taxifolin sulphate 2 \\
\hline$M 62^{\mathrm{c}}$ & 42.375 & $\mathrm{C}_{16} \mathrm{H}_{14} \mathrm{O}_{11} \mathrm{~S}$ & {$[\mathrm{M}-\mathrm{H}]^{-}$} & 413.0198 & 413.0184 & 3.39 & 10 & $\Delta$ & - & - & Level 3 & Hydroxylated methyl taxifolin sulphate 3 \\
\hline M63 ${ }^{\mathrm{c}}$ & 42.660 & $\mathrm{C}_{16} \mathrm{H}_{14} \mathrm{O}_{11} \mathrm{~S}$ & {$[\mathrm{M}-\mathrm{H}]^{-}$} & 413.0191 & 413.0184 & 1.69 & 10 & - & $\Delta$ & - & Level 3 & Hydroxylated methyl taxifolin sulphate 4 \\
\hline $\mathrm{M} 64^{\mathrm{c}}$ & 55.808 & $\mathrm{C}_{16} \mathrm{H}_{14} \mathrm{O}_{9}$ & {$[\mathrm{M}-\mathrm{H}]^{-}$} & 349.0580 & 349.0565 & 4.30 & 10 & $\Delta$ & - & $\Delta$ & Level 3 & Methylated and dihydroxylated taxifolin 1 \\
\hline $\mathrm{M} 65^{\mathrm{c}}$ & 56.608 & $\mathrm{C}_{16} \mathrm{H}_{14} \mathrm{O}_{9}$ & {$[\mathrm{M}-\mathrm{H}]^{-}$} & 349.0551 & 349.0565 & -4.01 & 10 & - & - & - & Level 3 & Methylated and dihydroxylated taxifolin 2 \\
\hline M66 ${ }^{\mathrm{c}}$ & 17.170 & $\mathrm{C}_{22} \mathrm{H}_{22} \mathrm{O}_{15}$ & {$[\mathrm{M}-\mathrm{H}]^{-}$} & 525.0865 & 525.0886 & -4.00 & 12 & - & $\Delta$ & - & Level 3 & Methylated and dihydroxylated taxifolin glucuronide 1 \\
\hline M67 ${ }^{\mathrm{c}}$ & 17.887 & $\mathrm{C}_{22} \mathrm{H}_{22} \mathrm{O}_{15}$ & {$[\mathrm{M}-\mathrm{H}]^{-}$} & 525.0908 & 525.0886 & 4.19 & 12 & - & $\Delta$ & - & Level 3 & Methylated and dihydroxylated taxifolin glucuronide 2 \\
\hline $\mathrm{M} 68^{\mathrm{c}}$ & 18.637 & $\mathrm{C}_{22} \mathrm{H}_{22} \mathrm{O}_{15}$ & {$[\mathrm{M}-\mathrm{H}]^{-}$} & 525.0890 & 525.0886 & 0.76 & 12 & - & $\Delta$ & - & Level 3 & Methylated and dihydroxylated taxifolin glucuronide 3 \\
\hline M69 ${ }^{\mathrm{C}}$ & 19.178 & $\mathrm{C}_{22} \mathrm{H}_{22} \mathrm{O}_{15}$ & {$[\mathrm{M}-\mathrm{H}]^{-}$} & 525.0911 & 525.0886 & 4.76 & 12 & - & $\Delta$ & - & Level 3 & Methylated and dihydroxylated taxifolin glucuronide 4 \\
\hline
\end{tabular}


Table 1. Cont.

\begin{tabular}{|c|c|c|c|c|c|c|c|c|c|c|c|c|}
\hline No. & $t_{R}(\min )$ & Formula & Ion & Meas. $\mathrm{m} / \mathrm{z}$ & Pred. $m / z$ & Diff (ppm) & DBE & Urine & Plasma & Faeces & Identification Level & Identification \\
\hline & & & & & \multicolumn{8}{|c|}{ Metabolites having the aglycone of quercetin(M70-M103); eight bioactive metabolites } \\
\hline M70 a,d & 58.150 & $\mathrm{C}_{15} \mathrm{H}_{10} \mathrm{O}_{7}$ & {$[\mathrm{M}-\mathrm{H}]^{-}$} & 301.0350 & 301.0354 & -1.33 & 11 & $\Delta$ & - & $\Delta$ & Level 2 & Quercetin \\
\hline M71 d & 51.583 & $\mathrm{C}_{15} \mathrm{H}_{10} \mathrm{O}_{10} \mathrm{~S}$ & {$[\mathrm{M}-\mathrm{H}]^{-}$} & 380.9933 & 380.9922 & 0.26 & 11 & 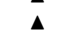 & - & $=$ & Level 2 & Quercetin-5-O-sulphate \\
\hline M72d & 52.647 & $\mathrm{C}_{15} \mathrm{H}_{10} \mathrm{O}_{10} \mathrm{~S}$ & {$[\mathrm{M}-\mathrm{H}]^{-}$} & 380.9932 & 380.9922 & 2.89 & 11 & 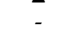 & - & - & Level 2 & Quercetin-7-O-sulphate \\
\hline M73 a,d & 56.300 & $\mathrm{C}_{15} \mathrm{H}_{10} \mathrm{O}_{10} \mathrm{~S}$ & {$[\mathrm{M}-\mathrm{H}]^{-}$} & 380.9922 & 380.9922 & 0.00 & 11 & $\Delta$ & - & $\Delta$ & Level 2 & Quercetin-4'-O-sulphate \\
\hline M74 a,d & 57.033 & $\mathrm{C}_{15} \mathrm{H}_{10} \mathrm{O}_{10} \mathrm{~S}$ & {$[\mathrm{M}-\mathrm{H}]^{-}$} & 380.9932 & 380.9922 & 2.62 & 11 & $\Delta$ & - & $\Delta$ & Level 2 & Quercetin-3'-O-sulphate \\
\hline M75 a,d & 58.173 & $\mathrm{C}_{15} \mathrm{H}_{10} \mathrm{O}_{10} \mathrm{~S}$ & {$[\mathrm{M}-\mathrm{H}]^{-}$} & 380.9937 & 380.9922 & 3.94 & 11 & & - & - & Level 2 & Quercetin-3-O-sulphate \\
\hline M76 ${ }^{\mathrm{a}}$ & 37.542 & $\mathrm{C}_{21} \mathrm{H}_{18} \mathrm{O}_{13}$ & {$[\mathrm{M}-\mathrm{H}]^{-}$} & 477.0688 & 477.0675 & 2.72 & 13 & $\Delta$ & - & - & Level 2 & Quercetin glucuronide \\
\hline M77 & 40.727 & $\mathrm{C}_{21} \mathrm{H}_{18} \mathrm{O}_{16} \mathrm{~S}$ & {$[\mathrm{M}-\mathrm{H}]^{-}$} & 557.0252 & 557.0243 & 1.62 & 13 & 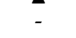 & $\Delta$ & - & Level 2 & Quercetin glucuronide sulphate 1 \\
\hline M78 & 41.068 & $\mathrm{C}_{21} \mathrm{H}_{18} \mathrm{O}_{16} \mathrm{~S}$ & {$[\mathrm{M}-\mathrm{H}]^{-}$} & 557.0268 & 557.0243 & 4.49 & 13 & - & $\Delta$ & - & Level 2 & Quercetin glucuronide sulphate 2 \\
\hline M79 & 41.443 & $\mathrm{C}_{21} \mathrm{H}_{18} \mathrm{O}_{16} \mathrm{~S}$ & {$[\mathrm{M}-\mathrm{H}]^{-}$} & 557.0269 & 557.0243 & 4.67 & 13 & - & $\Delta$ & - & Level 2 & Quercetin glucuronide sulphate 3 \\
\hline$M^{2} 0^{a, d}$ & 65.417 & $\mathrm{C}_{16} \mathrm{H}_{12} \mathrm{O}_{7}$ & {$[\mathrm{M}-\mathrm{H}]^{-}$} & 315.0503 & 315.0510 & -2.22 & 11 & $\Delta$ & - & $\Delta$ & Level 2 & $\begin{array}{l}\text { Isorhamnetin } \\
\text { S sulco }\end{array}$ \\
\hline M81 d & 48.633 & $\mathrm{C}_{16} \mathrm{H}_{12} \mathrm{O}_{10} \mathrm{~S}$ & {$[\mathrm{M}-\mathrm{H}]^{-}$} & 395.0081 & 395.0078 & 0.76 & 11 & $\Delta$ & $\Delta$ & - & Level 2 & Isorhamnetin-5-O-sulphate \\
\hline M82 ${ }^{d}$ & 56.917 & $\mathrm{C}_{16} \mathrm{H}_{12} \mathrm{O}_{10} \mathrm{~S}$ & {$[\mathrm{M}-\mathrm{H}]^{-}$} & 395.0082 & 395.0078 & 1.01 & 11 & 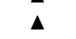 & $=$ & $\Delta$ & Level 2 & Isorhamnetin-7-O-sulphate \\
\hline$M 83^{a, d}$ & 58.042 & $\mathrm{C}_{16} \mathrm{H}_{12} \mathrm{O}_{10} \mathrm{~S}$ & {$[\mathrm{M}-\mathrm{H}]^{-}$} & 395.0085 & 395.0078 & 1.77 & 11 & $\bar{\Delta}$ & $\Delta$ & $\bar{\Delta}$ & Level 2 & Isorhamnetin-3-O-sulphate \\
\hline M84 ${ }^{\mathrm{d}}$ & 58.922 & $\mathrm{C}_{16} \mathrm{H}_{12} \mathrm{O}_{10} \mathrm{~S}$ & {$[\mathrm{M}-\mathrm{H}]^{-}$} & 395.0082 & 395.0078 & 1.01 & 11 & & $\Delta$ & & Level 2 & Isorhamnetin-4'-O-sulphate \\
\hline M85 $5^{a}$ & 48.308 & $\mathrm{C}_{16} \mathrm{H}_{12} \mathrm{O}_{13} \mathrm{~S}_{2}$ & {$[\mathrm{M}-\mathrm{H}]^{-}$} & 474.9658 & 474.9647 & 2.32 & 11 & $\Delta$ & $\Delta$ & - & Level 2 & $\begin{array}{l}\text { Isorhamnetin disulphate } \\
\text { S }\end{array}$ \\
\hline M86 ${ }^{\mathrm{d}}$ & 49.212 & $\mathrm{C}_{22} \mathrm{H}_{20} \mathrm{O}_{13}$ & {$[\mathrm{M}-\mathrm{H}]^{-}$} & 491.0852 & 491.0831 & 4.28 & 13 & - & $\Delta$ & - & Level 2 & Isorhamnetin-4'- $\mathbf{t}^{\prime}$-glucuronide \\
\hline M87 $7^{d}$ & 50.428 & $\mathrm{C}_{22} \mathrm{H}_{20} \mathrm{O}_{13}$ & {$[\mathrm{M}-\mathrm{H}]^{-}$} & 491.0836 & 491.0831 & 1.02 & 13 & - & $\Delta$ & - & Level 2 & Isorhamnetin-7-O-glucuronide \\
\hline M88 & 40.143 & $\mathrm{C}_{22} \mathrm{H}_{20} \mathrm{O}_{16} \mathrm{~S}$ & {$[\mathrm{M}-\mathrm{H}]^{-}$} & 571.0381 & 571.0399 & -3.15 & 13 & - & $\Delta$ & - & Level 2 & Isorhamnetin glucuronide sulphate 1 \\
\hline M89 & 41.118 & $\mathrm{C}_{22} \mathrm{H}_{20} \mathrm{O}_{16} \mathrm{~S}$ & {$[\mathrm{M}-\mathrm{H}]^{-}$} & 571.0413 & 571.0399 & 2.45 & 13 & - & $\Delta$ & - & Level 2 & Isorhamnetin glucuronide sulphate 2 \\
\hline M90 & 44.673 & $\mathrm{C}_{22} \mathrm{H}_{20} \mathrm{O}_{16} \mathrm{~S}$ & {$[\mathrm{M}-\mathrm{H}]^{-}$} & 571.0395 & 571.0399 & -0.70 & 13 & - & $\Delta$ & - & Level 2 & Isorhamnetin glucuronide sulphate 3 \\
\hline M91 & 45.392 & $\mathrm{C}_{22} \mathrm{H}_{20} \mathrm{O}_{16} \mathrm{~S}$ & {$\left[\mathrm{M}-\mathrm{H}^{-}\right.$} & 571.0419 & 571.0399 & 3.50 & 13 & - & - & - & Level 2 & Isorhamnetin glucuronide sulphate 4 \\
\hline M92 & 27.987 & $\mathrm{C}_{15} \mathrm{H}_{10} \mathrm{O}_{11} \mathrm{~S}$ & {$[\mathrm{M}-\mathrm{H}]^{-}$} & 396.9882 & 396.9871 & 2.77 & 11 & - & $\Delta$ & - & Level 2 & Hydroxylated quercetin sulphate 1 \\
\hline M93 & 28.487 & $\mathrm{C}_{15} \mathrm{H}_{10} \mathrm{O}_{11} \mathrm{~S}$ & {$[\mathrm{M}-\mathrm{H}]^{-}$} & 396.9868 & 396.9871 & -0.76 & 11 & - & $\Delta$ & - & Level 2 & Hydroxylated quercetin sulphate 2 \\
\hline M94 & 29.028 & $\mathrm{C}_{15} \mathrm{H}_{10} \mathrm{O}_{11} \mathrm{~S}$ & {$[\mathrm{M}-\mathrm{H}]^{-}$} & 396.9876 & 396.9871 & 1.26 & 11 & - & $\Delta$ & - & Level 2 & Hydroxylated quercetin sulphate 3 \\
\hline M95 & 15.930 & $\mathrm{C}_{21} \mathrm{H}_{18} \mathrm{O}_{14}$ & {$[\mathrm{M}-\mathrm{H}]^{-}$} & 493.0642 & 493.0624 & 3.65 & 13 & - & $\Delta$ & - & Level 2 & Hydroxylated quercetin glucuronide 1 \\
\hline M96 & 17.720 & $\mathrm{C}_{21} \mathrm{H}_{18} \mathrm{O}_{14}$ & {$[\mathrm{M}-\mathrm{H}]^{-}$} & 493.0601 & 493.0624 & -4.66 & 13 & - & $\Delta$ & - & Level 2 & Hydroxylated quercetin glucuronide 2 \\
\hline M97 & 39.160 & $\mathrm{C}_{16} \mathrm{H}_{12} \mathrm{O}_{11} \mathrm{~S}$ & {$[\mathrm{M}-\mathrm{H}]^{-}$} & 411.0022 & 411.0028 & -1.46 & 11 & - & $\Delta$ & - & Level 2 & Hydroxylated isorhamnetin sulphate 1 \\
\hline M98 & 39.710 & $\mathrm{C}_{16} \mathrm{H}_{12} \mathrm{O}_{11} \mathrm{~S}$ & {$[\mathrm{M}-\mathrm{H}]^{-}$} & 411.0043 & 411.0028 & 3.65 & 11 & - & $\Delta$ & - & Level 2 & Hydroxylated isorhamnetin sulphate 2 \\
\hline M99 & 40.193 & $\mathrm{C}_{16} \mathrm{H}_{12} \mathrm{O}_{11} \mathrm{~S}$ & {$[\mathrm{M}-\mathrm{H}]^{-}$} & 411.0039 & 411.0028 & 2.68 & 11 & - & $\bar{\Delta}$ & - & Level 2 & Hydroxylated isorhamnetin sulphate 3 \\
\hline M100 & 59.017 & $\mathrm{C}_{16} \mathrm{H}_{12} \mathrm{O}_{11} \mathrm{~S}$ & {$[\mathrm{M}-\mathrm{H}]^{-}$} & 411.0030 & 411.0028 & 0.49 & 11 & $\Delta$ & $\bar{\Delta}$ & - & Level 2 & \\
\hline M101 & 25.103 & $\mathrm{C}_{22} \mathrm{H}_{20} \mathrm{O}_{14}$ & {$[\mathrm{M}-\mathrm{H}]^{-}$} & 507.0790 & 507.0780 & 1.97 & 13 & - & $\Delta$ & - & Level 2 & Hydroxylated isorhamnetin glucuronide 1 \\
\hline & 25.728 & $\mathrm{C}_{22} \mathrm{H}_{20} \mathrm{O}_{14}$ & {$[\mathrm{M}-\mathrm{H}]^{-}$} & 507.0758 & 507.0780 & -4.34 & 13 & - & $\Delta$ & - & Level 2 & Hydroxylated isorhamnetin glucuronide 2 \\
\hline \multirow[t]{2}{*}{ M103 } & 26.570 & $\mathrm{C}_{22} \mathrm{H}_{20} \mathrm{O}_{14}$ & {$[\mathrm{M}-\mathrm{H}]^{-}$} & 507.0805 & 507.0780 & 4.93 & 13 & - & $\Delta$ & - & Level 2 & Hydroxylated isorhamnetin glucuronide 3 \\
\hline & & & & & \multicolumn{8}{|c|}{ Metabolites having the aglycone of dehydroxylated taxifolin (M104-M112); two bioactive metabolites } \\
\hline M104 a,b,d & 40.733 & $\mathrm{C}_{15} \mathrm{H}_{12} \mathrm{O}_{6}$ & {$[\mathrm{M}-\mathrm{H}]^{-}$} & 287.0557 & 287.0561 & -1.39 & 10 & - & - & $\Delta$ & Level 2 & Eriodictyol \\
\hline M105 a,b,d & 49.442 & $\mathrm{C}_{15} \mathrm{H}_{12} \mathrm{O}_{6}$ & {$[\mathrm{M}-\mathrm{H}]^{-}$} & 287.0555 & 287.0561 & -2.09 & 10 & $\Delta$ & - & $\Delta$ & Level 2 & Dihydrokaempferol \\
\hline M106 d & 37.325 & $\mathrm{C}_{15} \mathrm{H}_{12} \mathrm{O}_{9} \mathrm{~S}$ & {$[\mathrm{M}-\mathrm{H}]^{-}$} & 367.0128 & 367.0129 & -0.27 & 10 & 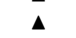 & - & 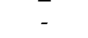 & Level 2 & Eriodictyol-7-O-sulphate \\
\hline M107 $^{\text {d }}$ & 37.708 & $\mathrm{C}_{15} \mathrm{H}_{12} \mathrm{O}_{9} \mathrm{~S}$ & {$[\mathrm{M}-\mathrm{H}]^{-}$} & 367.0144 & 367.0129 & 4.09 & 10 & $\Delta$ & - & $\Delta$ & Level 2 & Dihydrokaempferol-7-O-sulphate \\
\hline M108 $^{d}$ & 38.200 & $\mathrm{C}_{15} \mathrm{H}_{12} \mathrm{O}_{9} \mathrm{~S}$ & {$[\mathrm{M}-\mathrm{H}]^{-}$} & 367.0144 & 367.0129 & 4.09 & 10 & $\Delta$ & - & $\Delta$ & Level 2 & Eriodictyol-3'-O-sulphate \\
\hline M109 $^{d}$ & 40.383 & $\mathrm{C}_{15} \mathrm{H}_{12} \mathrm{O}_{9} \mathrm{~S}$ & {$[\mathrm{M}-\mathrm{H}]^{-}$} & 367.0123 & 367.0129 & -1.63 & 10 & - & - & - & Level 2 & Dihydrokaempferol- $4^{\prime}-\mathrm{O}$-sulphate \\
\hline M110 & 28.045 & $\mathrm{C}_{21} \mathrm{H}_{20} \mathrm{O}_{12}$ & {$[\mathrm{M}-\mathrm{H}]^{-}$} & 463.0907 & 463.0882 & 5.40 & 12 & - & $\Delta$ & - & Level 2 & Dehydroxylated taxifolin glucuronide 1 \\
\hline M111 & 28.753 & $\mathrm{C}_{21} \mathrm{H}_{20} \mathrm{O}_{12}$ & {$[\mathrm{M}-\mathrm{H}]^{-}$} & 463.0856 & 463.0882 & -5.61 & 12 & - & $\Delta$ & - & Level 2 & Dehydroxylated taxifolin glucuronide 2 \\
\hline $\mathrm{M} 112 \mathrm{~d}^{\mathrm{d}}$ & 28.970 & $\mathrm{C}_{21} \mathrm{H}_{20} \mathrm{O}_{12}$ & {$[\mathrm{M}-\mathrm{H}]^{-}$} & 463.0888 & 463.0882 & 1.30 & 12 & - & $\bar{\Delta}$ & - & Level 2 & Dihydrokaempferol- $-4^{\prime}-\mathrm{O}$-glucuronide \\
\hline
\end{tabular}


Table 1. Cont.

\begin{tabular}{|c|c|c|c|c|c|c|c|c|c|c|c|c|}
\hline No. & $t_{\mathrm{R}}(\min )$ & Formula & Ion & Meas. $m / z$ & Pred. $m / z$ & Diff (ppm) & DBE & Urine & Plasma & Faeces & Identification Level & Identification \\
\hline & & & & & \multicolumn{8}{|c|}{ Metabolites formed through dehydration and glucuronidation (M113-M116); one bioactive metabolite } \\
\hline M113 a,d & 16.017 & $\mathrm{C}_{21} \mathrm{H}_{18} \mathrm{O}_{12}$ & {$\left[\mathrm{M}+\mathrm{NH}_{2}\right]^{-}$} & 478.1007 & 478.0991 & 3.35 & 13 & $\Delta$ & - & - & Level 2 & Luteolin-7-O-glucuronide \\
\hline M114 d & 16.525 & $\mathrm{C}_{21} \mathrm{H}_{18} \mathrm{O}_{12}$ & {$\left[\mathrm{M}+\mathrm{NH}_{2}\right]^{-}$} & 478.1007 & 478.0991 & 3.35 & 13 & $\Delta$ & - & - & Level 2 & Luteolin- $3^{\prime} / 4^{\prime}-O$-glucuronide \\
\hline$M_{115} \mathrm{~d}$ & 17.425 & $\mathrm{C}_{21} \mathrm{H}_{18} \mathrm{O}_{12}$ & {$\left[\mathrm{M}+\mathrm{NH}_{2}\right]^{-}$} & 478.1014 & 478.0991 & 4.81 & 13 & $\Delta$ & - & - & Level 2 & Luteolin-- $3^{\prime} / 4^{\prime}$-O-glucuronide \\
\hline \multirow[t]{2}{*}{ M116 } & 23.625 & $\mathrm{C}_{22} \mathrm{H}_{20} \mathrm{O}_{12}$ & {$\left[\mathrm{M}+\mathrm{NH}_{2}\right]^{-}$} & 492.1160 & 492.1147 & 2.64 & 13 & $\Delta$ & - & - & Level 2 & Methyl luteolin glucuronide \\
\hline & & & & & \multicolumn{8}{|c|}{ Metabolites having the aglycone of hydrogenated taxifolin (M117-M121) } \\
\hline M117 & 43.883 & $\mathrm{C}_{15} \mathrm{H}_{14} \mathrm{O}_{7}$ & {$[\mathrm{M}-\mathrm{H}]^{-}$} & 305.0652 & 305.0667 & -4.92 & 9 & $\Delta$ & - & $\Delta$ & Level 2 & Hydrogenated taxifolin \\
\hline M118 & 52.325 & $\mathrm{C}_{16} \mathrm{H}_{16} \mathrm{O}_{7}$ & {$[\mathrm{M}-\mathrm{H}]^{-}$} & 319.0813 & 319.0823 & -3.13 & 9 & $\Delta$ & - & $\Delta$ & Level 2 & Hydrogenated methyl taxifolin \\
\hline M119 & 38.567 & $\mathrm{C}_{15} \mathrm{H}_{14} \mathrm{O}_{10} \mathrm{~S}$ & {$[\mathrm{M}-\mathrm{H}]^{-}$} & 385.0224 & 385.0235 & -2.86 & 9 & - & - & $\Delta$ & Level 2 & Hydrogenated taxifolin sulphate 1 \\
\hline M120 & 43.433 & $\mathrm{C}_{15} \mathrm{H}_{14} \mathrm{O}_{10} \mathrm{~S}$ & {$[\mathrm{M}-\mathrm{H}]^{-}$} & 385.0224 & 385.0235 & -2.86 & 9 & - & - & $\Delta$ & Level 2 & $\begin{array}{l}\text { Hydrogenated taxifolin sulphate } 2 \\
\text { Hot }\end{array}$ \\
\hline \multirow[t]{2}{*}{ M121 } & 45.442 & $\mathrm{C}_{15} \mathrm{H}_{14} \mathrm{O}_{10} \mathrm{~S}$ & {$[\mathrm{M}-\mathrm{H}]^{-}$} & 385.0227 & 385.0235 & -2.08 & 9 & $\Delta$ & - & $\boldsymbol{\Delta}$ & Level 2 & Hydrogenated taxifolin sulphate 3 \\
\hline & & & & & \multicolumn{8}{|c|}{ Phenolic acid metabolites through ring cleavage (M122-M159); four bioactive metabolites } \\
\hline M122 a,b,d & 35.317 & $\mathrm{C}_{9} \mathrm{H}_{10} \mathrm{O}_{3}$ & {$[\mathrm{M}-\mathrm{H}]^{-}$} & 165.0555 & 165.0557 & -1.21 & 5 & - & - & $\Delta$ & Level 2 & 3/4-Hydroxyphenylpropionic acid \\
\hline M123 a,d & 35.917 & $\mathrm{C}_{9} \mathrm{H}_{10} \mathrm{O}_{3}$ & {$[\mathrm{M}-\mathrm{H}]^{-}$} & 165.0559 & 165.0557 & 1.21 & 5 & $\Delta$ & - & 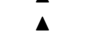 & Level 2 & 3/4-Hydroxyphenylpropionic acid \\
\hline M124 d & 21.712 & $\mathrm{C}_{9} \mathrm{H}_{10} \mathrm{O}_{6} \mathrm{~S}$ & {$[\mathrm{M}-\mathrm{H}]^{-}$} & 245.0132 & 245.0125 & 2.86 & 5 & $\Delta$ & - & - & Level 2 & 4-Hydroxyphenylpropionic acid sulphate \\
\hline M125 d & 23.683 & $\mathrm{C}_{9} \mathrm{H}_{10} \mathrm{O}_{6} \mathrm{~S}$ & {$[\mathrm{M}-\mathrm{H}]^{-}$} & 245.0133 & 245.0125 & 3.27 & 5 & $\Delta$ & $\Delta$ & - & Level 2 & 3-Hydroxyphenylpropionic acid sulphate \\
\hline M126 $^{d}$ & 23.787 & $\begin{array}{l}\mathrm{C}_{15} \mathrm{H}_{18} \mathrm{O}_{9} \\
\mathrm{C}_{2}\end{array}$ & {$[\mathrm{M}-\mathrm{H}]^{-}$} & 341.0866 & 341.0878 & $\begin{array}{c}3.27 \\
-1.76\end{array}$ & 7 & 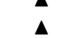 & - & - & Level 2 & 3/4-Hydroxyphenylpropionic acid glucuronide \\
\hline M127 d & 24.078 & $\mathrm{C}_{15} \mathrm{H}_{18} \mathrm{O}_{9}$ & {$[\mathrm{M}-\mathrm{H}]^{-}$} & 341.0891 & 341.0878 & 3.81 & 7 & $\Delta$ & - & - & $\begin{array}{l}\text { Level } 2 \\
\text { Level } 2\end{array}$ & $\begin{array}{l}\text { 3/4-Hydroxyphenyylpropionic acia glucuronide } \\
\text { 3/4id glucuronide }\end{array}$ \\
\hline M128 d & 22.325 & $\mathrm{C}_{9} \mathrm{H}_{8} \mathrm{O}_{6} \mathrm{~S}$ & {$[\mathrm{M}-\mathrm{H}]^{-}$} & 242.9969 & 242.9969 & 0.00 & 6 & $\Delta$ & - & - & Level 2 & $p / m$-Coumaric acid sulphate \\
\hline M129 d & 25.758 & $\mathrm{C}_{9} \mathrm{H}_{8} \mathrm{O}_{6} \mathrm{~S}$ & {$[\mathrm{M}-\mathrm{H}]^{-}$} & 242.9972 & 242.9969 & 1.23 & 6 & $\Delta$ & $\Delta$ & - & Level 2 & $p / m$-Coumaric acid sulphate \\
\hline M130 d & 27.067 & $\begin{aligned} & 9 \\
& \mathrm{C}_{9} \mathrm{H}_{8} \mathrm{O}_{6} \mathrm{O}_{6} \mathrm{~S}\end{aligned}$ & {$[\mathrm{M}-\mathrm{H}]^{-}$} & 242.9971 & 242.9969 & 0.82 & 6 & $\Delta$ & - & - & Level 2 & $p / m$-Coumaric acid sulphate \\
\hline M131 a,b,d & 16.490 & $\begin{array}{ll}918060^{\circ} \\
\mathrm{C} 8 \mathrm{H} 8 \mathrm{O}\end{array}$ & {$[\mathrm{M}-\mathrm{H}]^{-}$} & 167.0349 & 167.0350 & $\begin{array}{c}0.02 \\
-0.60\end{array}$ & 5 & - & - & $\Delta$ & Level 2 & Dihydroxyphenylacetic acid \\
\hline M132 d & 16.258 & $\mathrm{C}_{8} \mathrm{H}_{8} \mathrm{O}_{7} \mathrm{~S}$ & {$[\mathrm{M}-\mathrm{H}]^{-}$} & 246.9927 & 246.9918 & 3.64 & 5 & $\Delta$ & - & $\Delta$ & Level 2 & Dihydroxyphenylacetic acid sulfae 1 \\
\hline M133 ${ }^{d}$ & 15.800 & $\mathrm{C}_{8} \mathrm{H}_{8} \mathrm{O}_{7} \mathrm{~S}$ & {$[\mathrm{M}-\mathrm{H}]^{-}$} & 246.9927 & 246.9918 & 3.64 & 5 & & - & 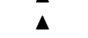 & Level 2 & Dihydroxyphenylacetic acid sulfae 2 \\
\hline M134 $^{\mathrm{d}}$ & 16.933 & $\mathrm{C}_{8} \mathrm{H}_{8} \mathrm{O}_{7} \mathrm{~S}$ & {$[\mathrm{M}-\mathrm{H}]^{-}$} & 246.9920 & 246.9918 & 0.81 & 5 & $\Delta$ & - & $\Delta$ & Level 2 & Dihydroxyphenylacetic acid sulfae 3 \\
\hline M135 d & 18.108 & $\mathrm{C}_{9} \mathrm{H}_{10} \mathrm{O}_{7} \mathrm{~S}$ & {$[\mathrm{M}-\mathrm{H}]^{-}$} & 261.0073 & 261.0074 & $\begin{array}{l}0.01 \\
-0.38\end{array}$ & 5 & $\Delta$ & - & - & Level 2 & $\begin{array}{l}\text { Homovanillic acid sulphate } \\
\text { Hond J }\end{array}$ \\
\hline M136 $^{\text {d }}$ & 22.508 & $\mathrm{C}_{9} \mathrm{H}_{10} \mathrm{O}_{4}$ & {$[\mathrm{M}-\mathrm{H}]^{-}$} & 181.0504 & 181.0506 & -1.10 & 5 & - & - & $\Delta$ & Level 2 & $\begin{array}{l}\text { Dihydrocaffeic acid } \\
\text { Did }\end{array}$ \\
\hline M137 d & 20.033 & $\mathrm{C}_{9} \mathrm{H}_{10} \mathrm{O}_{7} \mathrm{~S}$ & {$[\mathrm{M}-\mathrm{H}]^{-}$} & 261.0082 & 261.0074 & 3.07 & 5 & $\Delta$ & - & - & Level 2 & Dihydrocaffeic acid sulphate 1 \\
\hline M138 d & 20.942 & $\mathrm{C}_{9} \mathrm{H}_{10} \mathrm{O}_{7} \mathrm{~S}$ & {$[\mathrm{M}-\mathrm{H}]^{-}$} & 261.0084 & 261.0074 & 3.83 & 5 & $\Delta$ & - & - & Level 2 & Dihydrocaffeic acid sulphate 2 \\
\hline M139 d & 13.108 & $\mathrm{C}_{11} \mathrm{H}_{13} \mathrm{NO}_{5}$ & {$[\mathrm{M}-\mathrm{H}]^{-}$} & 238.0720 & 238.0721 & -0.42 & 6 & - & - & $\Delta$ & Level 2 & Caffeic acid acetamide 1 \\
\hline M140 d & 13.592 & $\begin{array}{ll}\mathrm{C}_{11} \mathrm{H}_{13} \mathrm{NO}_{5} \\
\mathrm{C}^{2}\end{array}$ & {$[\mathrm{M}-\mathrm{H}]^{-}$} & 238.0724 & 238.0721 & $\begin{array}{c}-.42 \\
1.26\end{array}$ & 6 & - & - & $\Delta$ & Level 2 & Caffeic acid acetamide 2 \\
\hline M141 d & 13.858 & $\mathrm{C}_{11} \mathrm{H}_{13} \mathrm{NO}_{5}$ & {$[\mathrm{M}-\mathrm{H}]^{-}$} & 238.0728 & 238.0721 & 2.94 & 6 & - & - & $\Delta$ & Level 2 & Caffeic acid acetamide 3 \\
\hline M142 d & 11.692 & $\mathrm{C}_{9} \mathrm{H}_{10} \mathrm{O}_{5}$ & {$[\mathrm{M}-\mathrm{H}]^{-}$} & 197.0461 & 197.0455 & 3.04 & 5 & $\Delta$ & - & $\Delta$ & Level 2 & 3-(3,4-Dihydroxyphenyl)-3-hydroxypropanoic acid \\
\hline M143 $^{\text {d }}$ & 12.658 & $\mathrm{C}_{9} \mathrm{H}_{10} \mathrm{O}_{5}$ & {$[\mathrm{M}-\mathrm{H}]^{-}$} & 197.0456 & 197.0455 & 0.51 & 5 & $\Delta$ & - & 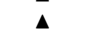 & Level 2 & 3-(3,4-Dihydroxyphenyl)-2-hydroxypropanoic acid \\
\hline M144 $^{\text {d }}$ & 12.700 & $\mathrm{C}_{9} \mathrm{H}_{10} \mathrm{O}_{8} \mathrm{~S}$ & {$[\mathrm{M}-\mathrm{H}]^{-}$} & 277.0024 & 277.0024 & 0.00 & 5 & 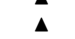 & - & 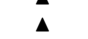 & Level 2 & $\begin{array}{l}\text { Caffeic acid hydrate sulphate } 1\end{array}$ \\
\hline M145 d & 13.433 & $\mathrm{C}_{9} \mathrm{H}_{10} \mathrm{O}_{8} \mathrm{~S}$ & {$[\mathrm{M}-\mathrm{H}]^{-}$} & 277.0025 & 277.0024 & 0.36 & 5 & $\Delta$ & - & $\Delta$ & Level 2 & Caffeic acid hydrate sulphate 2 \\
\hline$M_{146^{d}}$ & $\begin{array}{l}13.433 \\
22.667\end{array}$ & $\begin{array}{l}\mathrm{C}_{9} \mathrm{H}_{10} 08 \mathrm{~S} \\
\mathrm{C}_{10} \mathrm{H}_{12} \mathrm{O}_{7} \mathrm{~S}\end{array}$ & $\begin{array}{l}{[\mathrm{M}-\mathrm{H}]} \\
{[\mathrm{M}-\mathrm{H}]^{-}}\end{array}$ & 275.0236 & 275.0231 & $\begin{array}{l}1.36 \\
1.82\end{array}$ & $\begin{array}{l}5 \\
5\end{array}$ & 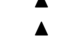 & - & - & Level 2 & Dihydrogen ferulic acid sulphate \\
\hline M146 d & 15.810 & $\begin{array}{l}10 \mathrm{C}_{12} \mathrm{C}_{12} \mathrm{O}_{7} \mathrm{~S} \\
\mathrm{C}_{10} \mathrm{H}_{12} \mathrm{O}_{8} \mathrm{~S}\end{array}$ & {$[\mathrm{M}-\mathrm{H}]^{-}$} & 291.0174 & 291.0180 & 3.78 & 5 & 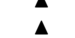 & - & - & Level 2 & Ferulic acid hydrate sulphate 1 \\
\hline M148 d & 16.233 & $\begin{array}{ll}10112 \\
\mathrm{C}_{10} \mathrm{H}_{12} \mathrm{O}_{8} \mathrm{~S}\end{array}$ & {$[\mathrm{M}-\mathrm{H}]^{-}$} & 291.0184 & 291.0180 & $\begin{array}{ll}1.10 \\
1.37\end{array}$ & 5 & $\Delta$ & - & - & $\begin{array}{l}\text { Level } 2 \\
\text { Level } 2\end{array}$ & $\begin{array}{l}\text { Ferulic accid hydrate esulphate } 1 \\
\text { Ferulic acid hydrate sulphate } 2\end{array}$ \\
\hline M149 & 25.208 & $\mathrm{C}_{7} \mathrm{H}_{8} \mathrm{O}_{5} \mathrm{~S}$ & {$[\mathrm{M}-\mathrm{H}]^{-}$} & 203.0021 & 203.0020 & 0.49 & 4 & $\Delta$ & - & - & Level 2 & Hydroxybenzyl alcohol sulphate \\
\hline${\mathrm{M} 150^{\mathrm{d}}}^{\mathrm{d}}$ & 29.025 & $\mathrm{C}_{13} \mathrm{H}_{16} \mathrm{O}_{8}$ & {$[\mathrm{M}-\mathrm{H}]^{-}$} & 299.0773 & 299.0772 & 0.33 & 6 & $\Delta$ & $\Delta$ & - & Level 2 & Hydroxybenzyl alcohol glucuronide 1 \\
\hline M151 d & 29.717 & $\mathrm{C}_{13} \mathrm{H}_{16} \mathrm{O}_{8}$ & {$[\mathrm{M}-\mathrm{H}]^{-}$} & 299.0771 & 299.0772 & -0.33 & 6 & $\Delta$ & $\Delta$ & - & Level 2 & Hydroxybenzyl alcohol glucuronide 2 \\
\hline M152 c,d & 18.795 & $\begin{array}{l}\mathrm{C}_{13} \mathrm{C}_{16} \mathrm{O}_{18} \mathrm{~S} \\
\mathrm{C}_{13} \mathrm{H}_{16} \mathrm{O}_{11}\end{array}$ & $\begin{array}{l}{[\mathrm{M}-\mathrm{H}]} \\
{[\mathrm{M}-\mathrm{H}]^{-}}\end{array}$ & 379.0336 & 379.0341 & $\begin{array}{l}-0.03 \\
-1.32\end{array}$ & 6 & - & 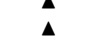 & - & Level 3 & Hydroxybenzyl alcohol glucuronide sulphate 1 \\
\hline M153 c,d & 21.095 & $\begin{array}{l}\mathrm{C}_{13} \mathrm{C}_{16} \mathrm{O}_{11} \mathrm{~S} \\
\mathrm{C}_{13} \mathrm{H}_{16} \mathrm{O}_{11} \mathrm{~S}\end{array}$ & {$[\mathrm{M}-\mathrm{H}]^{-}$} & 379.0337 & 379.0341 & $\begin{array}{l}-1.02 \\
-1.06\end{array}$ & 6 & - & $\Delta$ & - & Level 3 & Hydroxybenzyl alcohol glucuronide sulphate 2 \\
\hline M154 c,d & 33.083 & $\mathrm{C}_{8} \mathrm{H}_{10} \mathrm{O}_{5} \mathrm{~S}$ & {$[\mathrm{M}-\mathrm{H}]^{-}$} & 217.0168 & 217.0176 & $\begin{array}{l}-1.00 \\
-3.69\end{array}$ & 4 & $\Delta$ & - & - & $\begin{array}{l}\text { Level } 3 \\
\text { Level } 3\end{array}$ & $\begin{array}{l}\text { Hydroxbenenzyl aconol gucuronide supphate } 2 \\
\text { Methyl hydroxybenzyl alcohol sulphate } 1\end{array}$ \\
\hline M155 $5^{\mathrm{c}, \mathrm{d}}$ & 34.625 & $\mathrm{C}_{8} \mathrm{H}_{10} \mathrm{O}_{5} \mathrm{~S}$ & {$[\mathrm{M}-\mathrm{H}]^{-}$} & 217.0181 & 217.0176 & 2.30 & 4 & $\Delta$ & - & - & Level 3 & Methyl hydroxybenzyl alcohol sulphate 2 \\
\hline M156 d $^{d}$ & 17.512 & $\mathrm{C}_{7} \mathrm{H}_{6} \mathrm{O}_{6} \mathrm{~S}$ & {$[\mathrm{M}-\mathrm{H}]^{-}$} & 216.9822 & 216.9812 & 4.61 & 5 & - & $\Delta$ & - & Level 2 & 3/4-Hydroxy benzoic acid sulphate \\
\hline M156 d & 17.937 & $\begin{array}{l}\mathrm{C}_{7} \mathrm{H}_{6} \mathrm{U}_{6} \mathrm{O}_{6} \mathrm{~S} \\
\mathrm{C}^{2}\end{array}$ & $\begin{array}{l}{[\mathrm{M}-\mathrm{H}]} \\
{[\mathrm{M}-\mathrm{H}]^{-}}\end{array}$ & $\begin{array}{l}216.9822 \\
216.9810\end{array}$ & 216.9812 & $\begin{array}{c}4.61 \\
-0.92\end{array}$ & $\begin{array}{l}5 \\
5\end{array}$ & - & 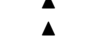 & - & $\begin{array}{l}\text { Level } 2 \\
\text { Level }\end{array}$ & 3/4-Hydroxy benzoic acid sulphate \\
\hline M158 ${ }^{d}$ & 30.987 & $\mathrm{C}_{8} \mathrm{H}_{8} \mathrm{O}_{7} \mathrm{~S}$ & {$[\mathrm{M}-\mathrm{H}]^{-}$} & $\begin{array}{l}240.9010 \\
246.9914\end{array}$ & 246.9918 & $\begin{array}{l}-0.92 \\
-1.62\end{array}$ & 5 & - & $\Delta$ & - & Level 2 & Vanillic acid sulphate \\
\hline M159 d & 31.978 & $\mathrm{C}_{8} \mathrm{H}_{8} \mathrm{O}_{7} \mathrm{~S}$ & {$[\mathrm{M}-\mathrm{H}]^{-}$} & 246.9909 & 246.9918 & $\begin{array}{l}-3.02 \\
-3.64\end{array}$ & 5 & - & $\Delta$ & - & Level 2 & Isovanillic acid sulphate \\
\hline
\end{tabular}


Table 1. Cont.

\begin{tabular}{|c|c|c|c|c|c|c|c|c|c|c|c|c|}
\hline No. & $t_{R}(\min )$ & Formula & Ion & Meas. $m / z$ & Pred. $m / z$ & Diff (ppm) & DBE & Urine & Plasma & Faeces & Identification Level & Identification \\
\hline & & & & & \multicolumn{8}{|c|}{ Metabolites formed through polymerization(M160-M191) } \\
\hline M160 & 61.342 & $\mathrm{C}_{31} \mathrm{H}_{24} \mathrm{O}_{13}$ & {$[\mathrm{M}-\mathrm{H}]^{-}$} & 603.1151 & 603.1144 & 1.16 & 20 & $\Delta$ & - & - & Level 2 & Dimer of taxiflolin and dehydroxylated methyl taxifolin \\
\hline${\mathrm{M} 161^{\mathrm{c}}}^{\mathrm{c}}$ & 55.533 & $\mathrm{C}_{31} \mathrm{H}_{24} \mathrm{O}_{14}$ & {$[\mathrm{M}-\mathrm{H}]^{-}$} & 619.1063 & 619.1093 & -4.85 & 20 & $\Delta$ & - & - & Level 3 & Dimer of taxiflolin and methyl taxifolin 1 \\
\hline${\mathrm{M} 162^{\mathrm{C}}}^{\mathrm{C}}$ & 60.600 & $\mathrm{C}_{31} \mathrm{H}_{24} \mathrm{O}_{14}$ & {$[\mathrm{M}-\mathrm{H}]^{-}$} & 619.1090 & 619.1093 & -0.48 & 20 & $\Delta$ & - & - & Level 3 & Dimer of taxiflolin and methyl taxifolin 2 \\
\hline $\mathrm{M} 163^{\mathrm{c}}$ & 64.608 & $\mathrm{C}_{32} \mathrm{H}_{26} \mathrm{O}_{14}$ & {$[\mathrm{M}-\mathrm{H}]^{-}$} & 633.1249 & 633.1250 & -0.16 & 20 & $\bar{\Delta}$ & - & - & Level 3 & Dimer of taxiflolin and dimethyl taxifolin \\
\hline M164 ${ }^{\mathrm{c}}$ & 56.025 & $\mathrm{C}_{31} \mathrm{H}_{24} \mathrm{O}_{17} \mathrm{~S}$ & {$[\mathrm{M}-\mathrm{H}]^{-}$} & 699.0699 & 699.0661 & 5.44 & 20 & $\bar{\Delta}$ & - & - & Level 3 & Dimer of taxiflolin and methyl taxifolin sulphate 1 \\
\hline M165 ${ }^{\mathrm{c}}$ & 56.750 & $\mathrm{C}_{31} \mathrm{H}_{24} \mathrm{O}_{17} \mathrm{~S}$ & {$[\mathrm{M}-\mathrm{H}]^{-}$} & 699.0671 & 699.0661 & 1.43 & 20 & $\bar{\Delta}$ & - & - & Level 3 & Dimer of taxiflolin and methyl taxifolin sulphate 2 \\
\hline M166 ${ }^{\mathrm{c}}$ & 60.817 & $\mathrm{C}_{31} \mathrm{H}_{24} \mathrm{O}_{17} \mathrm{~S}$ & {$[\mathrm{M}-\mathrm{H}]^{-}$} & 699.0678 & 699.0661 & 2.43 & 20 & $\bar{\Delta}$ & $\Delta$ & - & Level 3 & Dimer of taxiflolin and methyl taxifolin sulphate 3 \\
\hline M167 $\mathrm{c}$ & 59.725 & $\mathrm{C}_{32} \mathrm{H}_{26} \mathrm{O}_{17} \mathrm{~S}$ & {$[\mathrm{M}-\mathrm{H}]^{-}$} & 713.0844 & 713.0818 & 3.65 & 20 & $\bar{\Delta}$ & $=$ & - & Level 3 & Dimer of taxiflolin and dimethyl taxifolin sulphate 1 \\
\hline M168 ${ }^{\mathrm{c}}$ & 60.167 & $\mathrm{C}_{32} \mathrm{H}_{26} \mathrm{O}_{17} \mathrm{~S}$ & {$[\mathrm{M}-\mathrm{H}]^{-}$} & 713.0839 & 713.0818 & 2.94 & 20 & $\Delta$ & - & - & Level 3 & Dimer of taxiflolin and dimethyl taxifolin sulphate 2 \\
\hline M169 & 64.125 & $\mathrm{C}_{32} \mathrm{H}_{26} \mathrm{O}_{17} \mathrm{~S}$ & {$[\mathrm{M}-\mathrm{H}]^{-}$} & 713.0843 & 713.0818 & 3.51 & 20 & $\Delta$ & $\Delta$ & - & Level 3 & Dimer of taxiflolin and dimethyl taxifolin sulphate 3 \\
\hline M170 $^{c}$ & 60.650 & $\mathrm{C}_{32} \mathrm{H}_{26} \mathrm{O}_{13}$ & {$[\mathrm{M}-\mathrm{H}]^{-}$} & 617.1291 & 617.1301 & -1.62 & 20 & $\Delta$ & - & - & Level 3 & $\begin{array}{l}\text { Dimer of methyl taxiflolin and dehydroxylated methyl } \\
\text { taxifolin } 1\end{array}$ \\
\hline M171 ${ }^{\mathrm{c}}$ & 64.400 & $\mathrm{C}_{32} \mathrm{H}_{26} \mathrm{O}_{13}$ & {$[\mathrm{M}-\mathrm{H}]^{-}$} & 617.1311 & 617.1301 & 1.62 & 20 & $\Delta$ & - & - & Level 3 & $\begin{array}{l}\text { Dimer of methyl taxiflolin and dehydroxylated methyl } \\
\text { taxifolin } 2\end{array}$ \\
\hline M172 ${ }^{\mathrm{c}}$ & 64.925 & $\mathrm{C}_{32} \mathrm{H}_{26} \mathrm{O}_{13}$ & {$[\mathrm{M}-\mathrm{H}]^{-}$} & 617.1299 & 617.1301 & -0.32 & 20 & $\Delta$ & - & - & Level 3 & $\begin{array}{l}\text { Dimer of methyl taxiflolin and dehydroxylated methyl } \\
\text { taxifolin } 3\end{array}$ \\
\hline M173 ${ }^{\mathrm{c}}$ & 65.142 & $\mathrm{C}_{32} \mathrm{H}_{24} \mathrm{O}_{14}$ & {$[\mathrm{M}-\mathrm{H}]^{-}$} & 631.1093 & 631.1093 & 0.00 & 21 & $\Delta$ & - & - & Level 3 & Dimer of methyl quercetin and methyl taxifolin 1 \\
\hline${\mathrm{M} 174^{\mathrm{C}}}^{\mathrm{c}}$ & 66.142 & $\mathrm{C}_{32} \mathrm{H}_{24} \mathrm{O}_{14}$ & {$[\mathrm{M}-\mathrm{H}]^{-}$} & 631.1088 & 631.1093 & -0.79 & 21 & 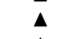 & - & - & Level 3 & Dimer of methyl quercetin and methyl taxifolin 2 \\
\hline${\mathrm{M} 175^{\mathrm{C}}}^{\mathrm{c}}$ & 68.517 & $\mathrm{C}_{32} \mathrm{H}_{24} \mathrm{O}_{14}$ & {$[\mathrm{M}-\mathrm{H}]^{-}$} & 631.1106 & 631.1093 & 2.06 & 21 & $\bar{\Delta}$ & - & - & Level 3 & Dimer of methyl quercetin and methyl taxifolin 3 \\
\hline M176 $^{\mathrm{c}}$ & 69.230 & $\mathrm{C}_{32} \mathrm{H}_{24} \mathrm{O}_{14}$ & {$[\mathrm{M}-\mathrm{H}]^{-}$} & 631.1105 & 631.1093 & 1.90 & 21 & $\Delta$ & - & - & Level 3 & Dimer of methyl quercetin and methyl taxifolin 4 \\
\hline M177 ${ }^{\mathrm{c}}$ & 64.550 & $\mathrm{C}_{33} \mathrm{H}_{28} \mathrm{O}_{13}$ & {$[\mathrm{M}-\mathrm{H}]^{-}$} & 631.1435 & 631.1457 & -3.49 & 20 & $\Delta$ & - & - & Level 3 & $\begin{array}{l}\text { Dimer of methyl taxiflolin and dehydroxylated } \\
\text { dimethyl taxifolin } 1\end{array}$ \\
\hline M178 $^{\mathrm{c}}$ & 67.408 & $\mathrm{C}_{33} \mathrm{H}_{28} \mathrm{O}_{13}$ & {$[\mathrm{M}-\mathrm{H}]^{-}$} & 631.1482 & 631.1457 & 3.96 & 20 & $\Delta$ & - & - & Level 3 & $\begin{array}{l}\text { Dimer of methyl taxiflolin and dehydroxylated } \\
\text { dimethyl taxifolin } 2\end{array}$ \\
\hline M179 ${ }^{\mathrm{c}}$ & 67.633 & $\mathrm{C}_{33} \mathrm{H}_{28} \mathrm{O}_{13}$ & {$[\mathrm{M}-\mathrm{H}]^{-}$} & 631.1488 & 631.1457 & 4.91 & 20 & $\Delta$ & - & - & Level 3 & $\begin{array}{l}\text { Dimer of methyl taxiflolin and dehydroxylated } \\
\text { dimethyl }\end{array}$ \\
\hline M180 ${ }^{\mathrm{c}}$ & 59.138 & $\mathrm{C}_{32} \mathrm{H}_{26} \mathrm{O}_{14}$ & {$[\mathrm{M}-\mathrm{H}]^{-}$} & 633.1257 & 633.1250 & 1.11 & 20 & $\Delta$ & - & - & Level 3 & Dimer of methyl taxiflolin and methyl taxifolin 1 \\
\hline $\mathrm{M}_{181^{\mathrm{c}}}{ }^{\mathrm{c}}$ & 63.783 & $\mathrm{C}_{32} \mathrm{H}_{26} \mathrm{O}_{14}$ & {$[\mathrm{M}-\mathrm{H}]^{-}$} & 633.1252 & 633.1250 & 0.32 & 20 & $\Delta$ & $\Delta$ & - & Level 3 & Dimer of methyl taxiflolin and methyl taxifolin 2 \\
\hline${\mathrm{M} 182^{\mathrm{C}}}^{\mathrm{C}}$ & 69.755 & $\mathrm{C}_{33} \mathrm{H}_{26} \mathrm{O}_{14}$ & {$[\mathrm{M}-\mathrm{H}]^{-}$} & 645.1243 & 645.1250 & -1.09 & 21 & $\Delta$ & - & - & Level 3 & Dimer of methyl taxiflolin and dimethyl quercetin 1 \\
\hline${\mathrm{M} 183^{\mathrm{c}}}^{\mathrm{c}}$ & 71.097 & $\mathrm{C}_{33} \mathrm{H}_{26} \mathrm{O}_{14}$ & {$[\mathrm{M}-\mathrm{H}]^{-}$} & 645.1252 & 645.1250 & 0.31 & 21 & $\Delta$ & - & - & Level 3 & Dimer of methyl taxiflolin and dimethyl quercetin 2 \\
\hline M184 ${ }^{\mathrm{c}}$ & 62.067 & $\mathrm{C}_{33} \mathrm{H}_{28} \mathrm{O}_{14}$ & {$[\mathrm{M}-\mathrm{H}]^{-}$} & 647.1432 & 647.1406 & 4.02 & 20 & $\Delta$ & - & - & Level 3 & Dimer of methyl taxiflolin and dimethyl taxifolin 1 \\
\hline M185 c & 62.600 & $\mathrm{C}_{33} \mathrm{H}_{28} \mathrm{O}_{14}$ & {$[\mathrm{M}-\mathrm{H}]^{-}$} & 647.1420 & 647.1406 & 2.16 & 20 & $\Delta$ & - & - & Level 3 & Dimer of methyl taxiflolin and dimethyl taxifolin 2 \\
\hline M186 ${ }^{\mathrm{C}}$ & 62.917 & $\mathrm{C}_{33} \mathrm{H}_{28} \mathrm{O}_{14}$ & {$[\mathrm{M}-\mathrm{H}]^{-}$} & 647.1419 & 647.1406 & 2.01 & 20 & $\Delta$ & - & - & Level 3 & Dimer of methyl taxiflolin and dimethyl taxifolin 3 \\
\hline M187 ${ }^{\mathrm{c}}$ & 63.183 & $\mathrm{C}_{33} \mathrm{H}_{28} \mathrm{O}_{14}$ & {$[\mathrm{M}-\mathrm{H}]^{-}$} & 647.1406 & 647.1406 & 0.00 & 20 & $\Delta$ & - & - & Level 3 & Dimer of methyl taxiflolin and dimethyl taxifolin 4 \\
\hline M188 ${ }^{\mathrm{c}}$ & 66.483 & $\mathrm{C}_{33} \mathrm{H}_{28} \mathrm{O}$ & {$[\mathrm{M}-\mathrm{H}]^{-}$} & 647.1434 & 647.1406 & 4.33 & 20 & $\Delta$ & - & - & Level 3 & Dimer of methyl taxiflolin and dimethyl taxifolin 5 \\
\hline M189 ${ }^{\mathrm{c}}$ & 66.983 & $\mathrm{C}_{33} \mathrm{H}_{28} \mathrm{O}_{14}$ & {$[\mathrm{M}-\mathrm{H}]^{-}$} & 647.1405 & 647.1406 & -0.15 & 20 & $\Delta$ & $\Delta$ & - & Level 3 & Dimer of methyl taxiflolin and dimethyl taxifolin 6 \\
\hline M190 ${ }^{\mathrm{c}}$ & 70.430 & $\mathrm{C}_{33} \mathrm{H}_{28} \mathrm{O}_{14}$ & {$[\mathrm{M}-\mathrm{H}]^{-}$} & 647.1421 & 647.1406 & 2.32 & 20 & $\Delta$ & $=$ & - & Level 3 & Dimer of methyl taxiflolin and dimethyl taxifolin 7 \\
\hline M191 c & 63.958 & $\mathrm{C}_{32} \mathrm{H}_{26} \mathrm{O}_{16} \mathrm{~S}$ & {$[\mathrm{M}-\mathrm{H}]^{-}$} & 697.0891 & 697.0869 & 3.16 & 20 & $\Delta$ & - & - & Level 3 & $\begin{array}{l}\text { Dimer of methyl taxiflolin and dehydroxylated methyl } \\
\text { taxifolin sulphate }\end{array}$ \\
\hline
\end{tabular}

Abbreviations: $\boldsymbol{\Lambda}$, detected; -, undetected; $t_{R}$, retention time; ${ }^{a}$ bioactivite metabolites ${ }^{\mathrm{b}}{ }^{\text {known }}$ metabolites of taxifolin $;{ }^{\mathrm{c}}$ new compounds; ${ }^{\mathrm{d}}$ metabolites have specific structures. Among 191 metabolites, M32, M65, M72, M75, M91, M109 were identified from the small intestine. 
Table 2. Metabolic reactions forming 191 metabolites of taxifolin detected in rats.

\begin{tabular}{|c|c|c|c|c|c|c|c|c|c|c|c|c|c|c|}
\hline \multirow{3}{*}{ No. } & \multirow{3}{*}{ Amount } & \multicolumn{13}{|c|}{ Metabolic Reaction } \\
\hline & & \multicolumn{8}{|c|}{ Phase I } & \multicolumn{5}{|c|}{ Phase II } \\
\hline & & $-\mathrm{H}_{2} \mathrm{O}$ & $-\mathrm{OH}$ & $+\mathrm{OH}$ & $-2 \mathrm{H}$ & $+2 \mathrm{H}$ & $\mathrm{RC}$ & I & $\mathbf{P}$ & $\mathrm{CH}_{3}$ & $+\mathrm{SO}_{3} \mathrm{H}$ & +GlcUA & $+\mathrm{AA}^{\mathrm{c}}$ & $+\mathrm{AM}^{\mathrm{c}}$ \\
\hline M1, M2 & 2 & & & & & & & $\Delta$ & & & & & & \\
\hline M3-M11 & 9 & & & & & & & & & & $\Delta$ & & & \\
\hline M12-M15 & 4 & & & & & & & & & & $\Delta \Delta^{a}$ & & & \\
\hline M16 & 1 & & & & & & & & & & $\Delta$ & & $\Delta$ & \\
\hline M17-M25 & $\begin{array}{l}1 \\
9\end{array}$ & & & & & & & & & & 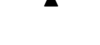 & $\Delta$ & 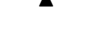 & \\
\hline M26-M32 & 7 & & & $\Delta$ & & & & & & & $\Delta$ & $\Delta$ & & \\
\hline M33-M36 & 4 & & & & & & & & & $\Delta$ & & & & \\
\hline M37-M46 & 10 & & & & & & & & & $\Delta$ & $\Delta$ & & & \\
\hline M47-M55 & 9 & & & & & & & & & $\Delta$ & & $\Delta$ & & \\
\hline M56, M57 & 2 & & & & & & & & & $\Delta$ & $\Delta$ & $\Delta$ & & \\
\hline M58, M59 & 2 & & & & & & & & & $\Delta$ & & & $\Delta$ & \\
\hline M60-M63 & 4 & & & $\Delta$ & & & & & & $\Delta$ & $\Delta$ & & 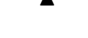 & \\
\hline M64, M65 & 2 & & & $\mathrm{~A}^{\mathrm{a}}$ & & & & & & 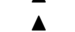 & & & & \\
\hline M66-M69 & 4 & & & $\mathbf{\Delta}^{\mathrm{a}}$ & & & & & & $\Delta$ & & $\Delta$ & & \\
\hline M70 & 1 & & & & $\Delta$ & & & & & & & & & \\
\hline M71-M75 & $\begin{array}{l}1 \\
5\end{array}$ & & & & $\Delta$ & & & & & & $\Delta$ & & & \\
\hline M76 & 1 & & & & 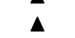 & & & & & & & $\Delta$ & & \\
\hline M77-M79 & 3 & & & & 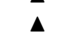 & & & & & $\Delta$ & & & & \\
\hline M80 & 1 & & & & 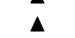 & & & & & 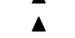 & $\Delta$ & & & \\
\hline M81-M84 & 4 & & & & $\bar{\Delta}$ & & & & & $\Delta$ & $\Delta$ & & & \\
\hline M85 & 1 & & & & $\Delta$ & & & & & $\Delta$ & $\Delta^{a}$ & $\Delta$ & & \\
\hline M86, M87 & 2 & & & & $\Delta$ & & & & & $\Delta$ & & $\Delta$ & & \\
\hline M88-M91 & 4 & & & & $\Delta$ & & & & & $\Delta$ & $\Delta$ & $\Delta$ & & \\
\hline M92-M94 & 3 & & & $\Delta$ & $\Delta$ & & & & & & $\bar{\Delta}$ & & & \\
\hline M95, M96 & 2 & & & $\Delta$ & $\Delta$ & & & & & & & $\Delta$ & & \\
\hline M97-M100 & 4 & & & $\Delta$ & $\Delta$ & & & & & $\Delta$ & $\Delta$ & 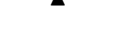 & & \\
\hline M101-M103 & 3 & & & $\Delta$ & $\Delta$ & & & & & $\Delta$ & & $\Delta$ & & \\
\hline M104, M105 & 2 & & $\Delta$ & & & & & & & & & & & \\
\hline M106-M109 & 4 & & $\Delta$ & & & & & & & & $\Delta$ & & & \\
\hline M110-M112 & 3 & & 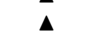 & & & & & & & & & $\Delta$ & & \\
\hline M113-M115 & 3 & $\Delta$ & & & & & & & & & & $\Delta$ & & \\
\hline M116 & 1 & $\Delta$ & & & & & & & & $\Delta$ & & $\Delta$ & & \\
\hline M117 & 1 & & & & & $\Delta$ & & & & 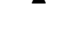 & & 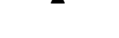 & & \\
\hline M118 & 1 & & & & & $\Delta$ & & & & $\Delta$ & & & & \\
\hline
\end{tabular}


Table 2. Cont.

\begin{tabular}{|c|c|c|c|c|c|c|c|c|c|c|c|c|c|c|}
\hline \multirow{3}{*}{ No. } & \multirow{3}{*}{ Amount } & \multicolumn{13}{|c|}{ Metabolic Reaction } \\
\hline & & \multicolumn{8}{|c|}{ Phase I } & \multicolumn{5}{|c|}{ Phase II } \\
\hline & & $-\mathrm{H}_{2} \mathrm{O}$ & $-\mathrm{OH}$ & $+\mathrm{OH}$ & $-2 \mathrm{H}$ & $+2 \mathrm{H}$ & RC & I & $\mathbf{P}$ & $\mathrm{CH}_{3}$ & $+\mathrm{SO}_{3} \mathrm{H}$ & + +GlcUA & $+\mathrm{AA}^{\mathrm{c}}$ & $+\mathrm{AM}^{\mathrm{C}}$ \\
\hline M119-M121 & 3 & & & & & $\Delta$ & & & & & $\Delta$ & & & \\
\hline M122, M123, & & & & & & & 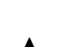 & & & & 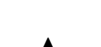 & & & \\
\hline $\begin{array}{l}\text { M131, M136, } \\
\text { M12, M13 }\end{array}$ & 6 & & & & & & $\Delta$ & & & & & & & \\
\hline M124, M125, & & & & & & & & & & & & & & \\
\hline M132-M135,M137, & 23 & & & & & & $\Delta$ & & & & $\Delta$ & & & \\
\hline $\begin{array}{l}\text { M138, 144-M149, } \\
\text { M154-M159 }\end{array}$ & & & & & & & & & & & & & & \\
\hline M126, M127, & 4 & & & & & & $\Delta$ & & & & & $\Delta$ & & \\
\hline M150, M151 & 3 & & & & & & $\Delta$ & & & & & & & 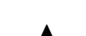 \\
\hline $\begin{array}{l}\text { M139-M141 } \\
\text { M152 M153 }\end{array}$ & 2 & & & & & & 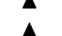 & & & & 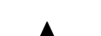 & $\mathbf{A}$ & & $\mathbf{A}$ \\
\hline $\begin{array}{l}\text { M152, M153 } \\
\text { M160 }\end{array}$ & 1 & & $\mathbf{\Delta}$ & & & & & & $\Delta$ & & & & & \\
\hline M161-M162 & 2 & & & & & & & & $\Delta$ & $\Delta$ & & & & \\
\hline M163, M180, M181 & 3 & & & & & & & & $\bar{\Delta}$ & $\Delta^{a}$ & & & & \\
\hline M164-M166 & 3 & & & & & & & & $\Delta$ & $\Delta$ & $\Delta$ & & & \\
\hline M167-M169 & 3 & & & & & & & & $\boldsymbol{\Delta}$ & $\mathbf{\Delta}^{\mathrm{a}}$ & $\mathbf{\Delta}$ & & & \\
\hline M170-M172 & 3 & & $\Delta$ & & & & & & $\Delta$ & $\mathbf{A}^{\mathrm{a}}$ & & & & \\
\hline M173-M176 & 4 & & & & $\Delta$ & & & & $\Delta$ & $\Delta{ }^{a}$ & & & & \\
\hline $\begin{array}{l}\text { M1777-M179 } \\
\text { M182, M183 }\end{array}$ & $\begin{array}{l}3 \\
2\end{array}$ & & & & $\Delta$ & & & & $\Delta$ & $\mathrm{A}^{\mathrm{b}}$ & & & & \\
\hline M184-M190 & 7 & & & & & & & & $\bar{\Delta}$ & $\mathrm{A}^{\mathrm{b}}$ & & & & \\
\hline M191 & ${ }_{1}^{1}$ & 4 & $\Delta$ & 20 & 4 & 5 & 38 & 2 & $\Delta$ & $\Delta^{a}$ & 103 & 57 & 3 & 3 \\
\hline & & 7 & & & & & & 2 & & & & & & \\
\hline
\end{tabular}

Abbreviations: $-\mathrm{H}_{2} \mathrm{O}$, dehydration; $-\mathrm{OH}$, dehydroxylation; $+\mathrm{OH}$, hydroxylation; $-2 \mathrm{H}$, dehydrogenation; $+2 \mathrm{H}$, hydrogenation; $\mathrm{RC}$, ring cleavage; I, isomerization; $\mathrm{P}$, polymerization $+\mathrm{CH}_{3}$, methylation; $+\mathrm{SO}_{3} \mathrm{H}$, sulphation; $+\mathrm{GlcUA}$, glucuronidation; $+\mathrm{AA}$, amino acid conjugation; $+\mathrm{AM}$, acetylamination. ${ }^{a}$ metabolic reaction repeated two times; ${ }^{\mathrm{b}}$ metabolic reaction repeated three times; ${ }^{\mathrm{C}}$ new metabolic reaction. $\mathbf{\Lambda}$, denoting the metabolic reaction is detected. 


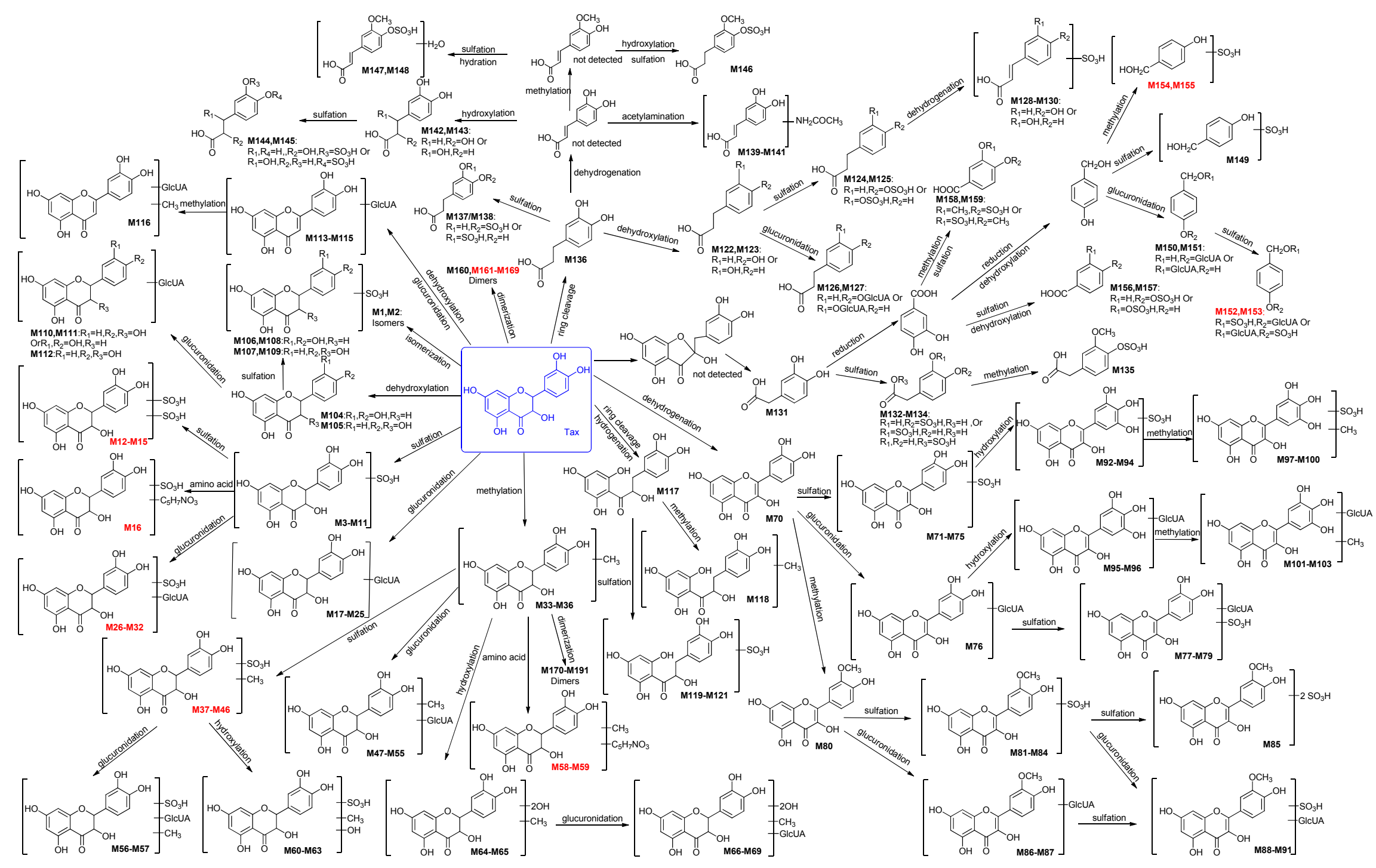

Figure 1. The proposed metabolic pathways of taxifolin in rats, with M1-M191 metabolites. The blue is taxifolin, the red shows new compound. 
Based on the ClogP rule (the smaller the ClogP value, the smaller the retention time value) [39], and considering that the main in vivo methylation metabolite of taxifolin is 3'-O-methyltaxifolin [30], M33 $\left(t_{R}=50.292\right.$, the relative peak area was the largest) was tentatively identified as $3^{\prime}$-O-methyl-taxifolin $(\mathrm{C} \log \mathrm{P}=1.21715) ; \mathrm{M} 34\left(\mathrm{t}_{\mathrm{R}}=51.350\right)$ as $4^{\prime}$-O-methyl-taxifolin $(\mathrm{C} \log \mathrm{P}=1.21715) ; \mathrm{M} 35\left(\mathrm{t}_{\mathrm{R}}=52.875\right)$ as 7-O-methyl-taxifolin ( $\operatorname{Clog} \mathrm{P}=1.29372)$; and $\mathrm{M} 36\left(\mathrm{t}_{\mathrm{R}}=53.592\right)$ as 3-O-methyl-taxifolin $(\mathrm{ClogP}=1.40805)$.

In NI MS² spectra of M37-M59, the same [aglycone $-\mathrm{H}^{-}(\mathrm{m} / \mathrm{z} 317.06)$ was observed with identical molecular formula and fragmentation behaviours to methyl taxifolin. We therefore deduced that they were conjugates of methyl taxifolin. M37-M46 were methyl taxifolin sulphates; M47-M55 were glucuronides of methyl taxifolin; M56-M57 were methyl taxifolin glucuronide sulphates and M58-M59 were identified as methyl taxifolin pyroglutamic acid conjugates similar to M16.

As for metabolites M60-M63 formed through methylation, hydroxylation and sulphation, the neutral loss of $79.95 \mathrm{Da}\left(\mathrm{SO}_{3}\right)$ was observed in the $\mathrm{MS}^{2}$ spectra of M60-M63 and the aglycone had the molecular formula of $\mathrm{C}_{16} \mathrm{H}_{14} \mathrm{O}_{8}$, one more oxygen atom (mass shifts of +15.99 ) than that of methyl taxifolin. We therefore deduced that these metabolites were sulphates of hydroxylated methyl taxifolin.

M64-M65 showed $[\mathrm{M}-\mathrm{H}]^{-}$at $\mathrm{m} / \mathrm{z}$ 349.06. Their molecular formulae were calculated to be $\mathrm{C}_{16} \mathrm{H}_{14} \mathrm{O}_{9}, 31.98 \mathrm{Da}(2 \mathrm{O})$ more than that of methyl taxifolin and resulting in their temporary identification as methylated and dihydroxyled taxifolin. M66-M69 showed $[\mathrm{M}-\mathrm{H}]^{-}$at $\mathrm{m} / \mathrm{z} 525.09$ and then yielded [aglycone $-\mathrm{H}]^{-}$at $\mathrm{m} / \mathrm{z} 349.06$ by neutral loss of $176.03 \mathrm{Da}$; the aglycones were identical to M64-M65. Hence, these metabolites were tentatively identified as glucuronides of methylated and dihydroxylated taxifolin.

\subsubsection{Identification of 34 Metabolites (M70-M103) Having the Aglycone of Quercetin}

In total, 34 metabolites having the aglycone of quercetin were found from the drug group, including quercetin, isorhamnetin, nine quercetin conjugates, 11 isorhamnetin conjugates and 12 conjugates of hydroxylated quercetin.

Metabolite M70 was formed through dehydrogenation. The $[\mathrm{M}-\mathrm{H}]^{-}$of $\mathbf{M 7 0}$ was at $m / z 301.0349$ $\left(\mathrm{C}_{15} \mathrm{H}_{9} \mathrm{O}_{7}\right)$, which is $2.01 \mathrm{Da}\left(\mathrm{H}_{2}\right)$ less than taxifolin, and the retention time and characteristic fragment ions were the same as those for the reference compound quercetin. M70 was thus determined to be quercetin.

In the NI MS ${ }^{2}$ spectra of M71-M79, the same [aglycone - $\left.\mathrm{H}\right]^{-}(\mathrm{m} / \mathrm{z}$ 301.04) was observed with identical molecular formula and fragmentation behaviour as quercetin. We therefore deduced that they were conjugates of quercetin. Based on characteristic neutral losses, M71-M75 were identified as quercetin sulphates. According to the $C \log \mathrm{P}$ rule, $\mathbf{M} 71\left(\mathrm{t}_{\mathrm{R}}=51.583\right)$ was quercetin-5-O-sulphate $(\mathrm{C} \log \mathrm{P}=-0.897894), \mathbf{M 7 2}\left(\mathrm{t}_{\mathrm{R}}=52.647\right)$ was quercetin-7-O-sulphate $(\mathrm{C} \log \mathrm{P}=0.00210607), \mathbf{M 7 3}$ $\left(t_{R}=56.3\right.$, relative peak area $\left.=378222\right)$ and $\mathbf{M 7 4}\left(t_{R}=57.033\right.$, relative peak area $\left.=3335213\right)$ were quercetin-3 $3^{\prime} / 4^{\prime}-O$-sulphate $(C \log P=0.0554161)$ and $\mathbf{M 7 5}\left(t_{R}=58.173\right)$ was quercetin-3-O-sulphate $(\mathrm{C} \log \mathrm{P}=0.160939)$. According to the literature [41], the favoured sulphation sites of quercetin are $3^{\prime}$ and 7-OH. The relative peak area of $\mathbf{M 7 4}$ was higher than that of M73, indicating that M74 was quercetin-3'-O-sulphate and M73 was quercetin-4'-O-sulphate. M76 was identified as quercetin glucuronide and M77-M79 were identified as quercetin glucuronide sulphates.

The molecular formula of $\mathbf{M 8 0}$ was calculated as $\mathrm{C}_{16} \mathrm{H}_{12} \mathrm{O}_{7}, 14.01 \mathrm{Da}\left(\mathrm{CH}_{2}\right)$ more than quercetin. Given that $3^{\prime}-\mathrm{OH}$ is the main methylation site of quercetin according to the literature [41], M80 was identified as $3^{\prime}$-O-methyl-quercetin (isorhamnetin). In the NI $\mathrm{MS}^{2}$ spectra of M81-M91, the same [aglycone $-\mathrm{H}]^{-}(\mathrm{m} / \mathrm{z} 315.05)$ was observed with identical molecular formula and fragmentation behaviour to isorhamnetin. Hence, these metabolites were considered as conjugates of isorhamnetin. M81-M84 were isorhamnetin sulphates. Based on the ClogP rule, M81 $\left(t_{R}=48.633\right)$ was isorhamnetin-5-O-sulphate $(C \log P=-0.452683), \mathbf{M} 82\left(t_{R}=56.917\right)$ was isorhamnetin-7-O-sulphate $(\mathrm{C} \log \mathrm{P}=0.447317), \mathbf{M} 83\left(\mathrm{t}_{\mathrm{R}}=58.042\right)$ was isorhamnetin-3-O-sulphate $(\mathrm{C} \log \mathrm{P}=0.605693)$ and M84 $\left(t_{R}=58.922\right)$ was the isorhamnetin- $4^{\prime}-O$-sulphate $(C \log P=0.631748)$. M85 was identified as isorhamnetin disulphate and M86-M87 were identified as glucuronides of isorhamnetin. According to 
the literature [41], M86-M87 was tentatively identified as isorhamnetin-4' /7-O-glucuronide. Based on the $C \log P$ rule, $\mathbf{M} 86\left(t_{R}=49.212\right)$ was isorhamnetin- $4^{\prime}-O$-glucuronide $(C \log \mathrm{P}=-0.133551)$ and $\mathbf{M} 87$ $\left(t_{R}=50.428\right)$ was isorhamnetin-7-O-glucuronide $(C \log P=0.0320181)$. M88-M91 were identified as isorhamnetin glucuronide sulphates.

In the NI MS ${ }^{2}$ spectra of M92-M96, the same aglycone $\left(\mathrm{C}_{15} \mathrm{H}_{10} \mathrm{O}_{8}\right), 15.99 \mathrm{Da}(\mathrm{O})$ more than quercetin, was observed; hence, they were identified as hydroxylated quercetins. In addition, we can deduce that they were conjugates of hydroxyquercerin. According to characteristic neutral losses, M92-M94 were identified as sulphates of hydroxylated quercetin. M95-M96 were glucuronides of hydroxylated quercetin.

In the NI MS² spectra of M97-M103, the same aglycone $\left(\mathrm{C}_{16} \mathrm{H}_{12} \mathrm{O}_{8}\right), 15.99 \mathrm{Da}(\mathrm{O})$ more than isorhamnetin, was observed, hence, it was identified as hydroxylated isorhamnetin. Furthermore, we deduced that these metabolites were conjugates of hydroxylated isorhamnetin. M97-M100 were tentatively identified as sulphates of hydroxylated isorhamnetin and M101-M103 were glucuronides of hydroxylated isorhamnetin.

\subsubsection{Identification of 9 Metabolites (M104-M112) Having the Aglycone of Dehydroxylated Taxifolin}

In total, nine metabolites including two dehydroxylated taxifolins, and seven conjugates of dehydroxylated taxifolin or isomers were identified.

The molecular formulae of M104 and M105 were calculated to be $\mathrm{C}_{15} \mathrm{H}_{12} \mathrm{O}_{6}$ and they were identified as dehydroxylated taxifolin when compared with taxifolin. The fragment ions at $\mathrm{m} / \mathrm{z} 137.0222$ $\left(0,2 \mathrm{~B}^{-}\right)$in the $\mathrm{MS}^{2}$ spectrum of M104 indicated that there were two hydroxyl groups linked to the B-ring, and that the A ring might have two hydroxyl groups based on $\mathrm{m} / \mathrm{z} 107.0174\left({ }^{0,4} \mathrm{~A}^{-}\right)$and $\mathrm{m} / \mathrm{z} 165.0205$ $\left({ }^{1,2} \mathrm{~A}^{-}\right)$. Therefore, M104 was tentatively identified as eriodictyol. The characteristic fragment ions of M105 at $m / z 269.0368\left(\left[\mathrm{M}-\mathrm{H}-\mathrm{H}_{2} \mathrm{O}\right]^{-}\right), m / z 259.0621\left([\mathrm{M}-\mathrm{H}-\mathrm{CO}]^{-}\right), m / z 243.0647([\mathrm{M}-\mathrm{H}-$ $\left.\left.\mathrm{CO}_{2}\right]^{-}\right), m / z 201.0554\left(\left[\mathrm{M}-\mathrm{H}-\mathrm{CO}_{2}-\mathrm{C}_{2} \mathrm{H}_{2} \mathrm{O}\right]^{-}\right), m / z 173.0683\left(\left[\mathrm{M}-\mathrm{H}-\mathrm{CO}-\mathrm{CO}_{2}-\mathrm{C}_{2} \mathrm{H}_{2} \mathrm{O}\right]^{-}\right)$ and $m / z 125.0290\left({ }^{1,4} \mathrm{~A}^{-}+2 \mathrm{H}\right)$ were consistent with the reference compound dihydrokaempferol. Hence, M105 was identified as dihydrokaempferol.

In the NI MS 2 spectra of M106-M112, the same [aglycone $-\mathrm{H}^{-}(m / z$ 287.05) with identical molecular formula and fragmentation behaviour to dehydroxylated taxifolin was observed, we therefore deduced that these were conjugates of dehydroxylated taxifolin. The characteristic fragment ions of the [aglycone $-\mathrm{H}^{-}$of M106 and M108 were the same as those of eriodictyol. Because the main sulphation sites were located at C- $3^{\prime}$ and C-7, and based on the ClogP rule, M106 $\left(t_{R}=37.325\right)$ was tentatively identified as eriodictyol-7-O-sulphate $(\mathrm{Clog} \mathrm{P}=0.224621)$ and $\mathrm{M108}\left(\mathrm{t}_{\mathrm{R}}=37.708\right)$ as eriodictyol-3'-O-sulphate $(\mathrm{C} \log \mathrm{P}=0.398051)$. The characteristic fragment ions of the [aglycone $-\mathrm{H}]^{-}$ of M107 and M109 were identical to those of dihydrokaempferol. Hence, M107 $\left(t_{R}=38.200\right)$ was dihydrokaempferol 7-O-sulphate $(\mathrm{ClogP}=-0.255279)$ and $\mathbf{M 1 0 9}\left(\mathrm{t}_{\mathrm{R}}=40.383\right)$ was dihydrokaempferol $4^{\prime}$-O-sulphate $(\mathrm{C} \log \mathrm{P}=-0.192048)$. M110-M112 yielded [aglycone $\left.-\mathrm{H}\right]^{-}$by neutral loss of 176.03 $\mathrm{Da}\left(\mathrm{C}_{6} \mathrm{H}_{8} \mathrm{O}_{6}\right)$, which suggested that M110-M112 were glucuronides of dehydroxylated taxifolin. The characteristic fragment ions of M112 were consistent with dihydrokaempferol, so M112 was considered to be dihydrokaempferol glucuronide.

\subsubsection{Identification of Four Metabolites (M113-M116) Formed through Dehydration and Glucuronidation}

Four metabolites were identified, including three luteolin glucuronides and one methyl luteolin glucuronide. M113-M115 showed $\left[\mathrm{M}+\mathrm{NH}_{3}-\mathrm{H}\right]^{-}$at $\mathrm{m} / \mathrm{z} 478.10$ (predicted to be $\mathrm{C}_{21} \mathrm{H}_{20} \mathrm{O}_{12} \mathrm{~N}$ ) in their HRMS data. The aglycone $+\mathrm{NH}_{3}-\mathrm{H}^{-}$was formed by the neutral loss of $176.03 \mathrm{Da}$ in the NI MS ${ }^{2}$ spectra and the aglycone had the molecular formula of $\mathrm{C}_{15} \mathrm{H}_{10} \mathrm{O}_{6}$, which is $18.01 \mathrm{Da}$ $\left(\mathrm{H}_{2} \mathrm{O}\right)$ less than taxifolin $\left(\mathrm{C}_{15} \mathrm{H}_{12} \mathrm{O}_{7}\right)$. The characteristic fragment ions of the aglycone were $\mathrm{m} / \mathrm{z} 217.06$ $\left(\left[\mathrm{M}-\mathrm{H}-\mathrm{C}_{3} \mathrm{O}_{2}\right]^{-}\right), m / z 175.03\left(\left[\mathrm{M}-\mathrm{H}-\mathrm{C}_{3} \mathrm{O}_{2}-\mathrm{C}_{2} \mathrm{H}_{2} \mathrm{O}\right]^{-}\right)$and $m / z 177.03\left({ }^{0,4} \mathrm{~B}^{-}\right)$, indicating that there were two hydroxyl groups linked to the A-ring and B-ring, respectively. Accordingly, the aglycone was considered as the dehydration metabolite of taxifolin and tentatively identified as 
luteolin. As a result, M113-M115 were glucuronides of luteolin. Because C-5 was not easily conjugated, the sites of glucuronidation were considered to be the hydroxyl groups of the $C-7,3^{\prime}$ and $4^{\prime}$ positions of luteolin. Based on the ClogP rule, M113 $\left(t_{R}=16.017\right)$ was luteolin-7-O-glucuronide $(C \log P=0.335925)$, and M114 $\left(t_{R}=16.583\right)$ and M115 $\left(t_{R}=17.483\right)$ were luteolin- $3^{\prime} / 4^{\prime}$-O-glucuronide $(\mathrm{ClogP}=0.188342)$. M116 showed $\left[\mathrm{M}+\mathrm{NH}_{3}-\mathrm{H}\right]^{-}$at $m / z 492.1165$ (predicted to be $\mathrm{C}_{22} \mathrm{H}_{23} \mathrm{O}_{12} \mathrm{~N}$ ) in the HRMS data. In the $\mathrm{MS}^{2}$ spectrum, the neutral loss of $176.03 \mathrm{Da}\left(\mathrm{C}_{6} \mathrm{H}_{8} \mathrm{O}_{6}\right)$ was observed and the aglycone was $14.01 \mathrm{Da}\left(\mathrm{CH}_{2}\right)$ more than luteolin. Hence, the aglycone was methyl luteolin, and M116 was identified as the glucuronide of methyl luteolin.

\subsubsection{Identification of Five Metabolites (M117-M121) Having the Aglycone of Hydrogenated Taxifolin}

In total, five metabolites including hydrogenated taxifolin, hydrogenated methyltaxifolin and three hydrogenated taxifolin sulphates were detected. M117 showed $[\mathrm{M}-\mathrm{H}]^{-}$at $m / z$ 305.0652, which was $2.01 \mathrm{Da}\left(\mathrm{H}_{2}\right)$ more than taxifolin, and the characteristic fragment ions were at $\mathrm{m} / z 287.0565$ $\left(\mathrm{C}_{15} \mathrm{H}_{11} \mathrm{O}_{6}\right), m / z 183.0309\left(\mathrm{C}_{8} \mathrm{H}_{7} \mathrm{O}_{5}\right), m / z 165.0249\left(\mathrm{C}_{8} \mathrm{H}_{5} \mathrm{O}_{4}\right), m / z 161.0287\left(\mathrm{C}_{9} \mathrm{H}_{5} \mathrm{O}_{3}\right)$ and $m / z 137.0301$ $\left(\mathrm{C}_{7} \mathrm{H}_{5} \mathrm{O}_{3}\right)$. Therefore, M117 was tentatively identified as a hydrogenated product. The molecular formula of M118 was calculated to be $\mathrm{C}_{16} \mathrm{H}_{16} \mathrm{O}_{7}$, which is $2.01 \mathrm{Da}\left(\mathrm{H}_{2}\right)$ more than that of methyltaxifolin; hence, M118 was identified as hydrogenated methyl taxifolin. M119-M121 yielded [aglycone $-\mathrm{H}]^{-}$at $\mathrm{m} / z 305.06$ by neutral loss of $79.95 \mathrm{Da}\left(\mathrm{SO}_{3}\right)$, indicating that they were hydrogenated taxifolin sulphates.

\subsubsection{Identification of 38 Metabolites (M122-M159) Having the Aglycone of Phenolic Acid Derivatives}

In total, 38 metabolites (four new compounds) having the aglycone of phenolic acid derivatives were found in the drug group, including phenolic acids and their conjugations.

Metabolites having the aglycone of hydroxyphenylpropanoic acid: M122-M130. The $[\mathrm{M}-\mathrm{H}]^{-}$ of M122-M123 were at $m / z$ 165.06, and characteristic fragment ions at $m / z 121.07$ and $m / z 119.04$ were observed in their MS $^{2}$ spectra. According to a previous report [42], we identified M122-M123 as 3/4-hydroxyphenylpropanoic acid. M124-M127 yielded [aglycone $-\mathrm{H}]^{-}$at $\mathrm{m} / \mathrm{z} 165.06$ by neutral loss of 79.95 Da or 176.03 Da. Hence, M124-M125 were identified as sulphates of hydroxyphenylpropanoic acid. M126-M127 were glucuronides of hydroxyphenylpropanoic acid. M128-M130 yielded [aglycone $-\mathrm{H}]^{-}$at $m / z 163.04\left(\mathrm{C}_{9} \mathrm{H}_{8} \mathrm{O}_{3}\right)$ by the loss of $\mathrm{SO}_{3}(79.96 \mathrm{Da})$ and produced characteristic fragment ions at $m / z 163.04$ (100.0) and $m / z 119.06$ (17.51). According to a previous report [39], we identified M128-M130 as $p / m$-coumaric acid sulphates.

Metabolites having the aglycone of dihydroxyphenylacetic acid: M131-M135. M131 showed $[\mathrm{M}-\mathrm{H}]^{-}$at $\mathrm{m} / \mathrm{z} 167.0349$ (predicted to be $\mathrm{C}_{8} \mathrm{H}_{7} \mathrm{O}_{4}$ ), and characteristic fragment ions at $\mathrm{m} / \mathrm{z} 123.0458$ were observed in NI MS² spectrum. According to a previous report [43], we identified M131 as dihydroxyphenylacetic acid, a known metabolite of taxifolin. M132-M134 yielded [aglycone $-\mathrm{H}]^{-}$at $\mathrm{m} / z 167.04$ by neutral loss of $79.95 \mathrm{Da}$, indicating that these were sulphates of dihydroxyphenylacetic acid. M135 showed $[\mathrm{M}-\mathrm{H}]^{-}$at $\mathrm{m} / z 261.0073$ and yielded [aglycone $\left.-\mathrm{H}\right]^{-}$at $\mathrm{m} / \mathrm{z} 181.0569$ by neutral loss of 79.95Da $\left(\mathrm{SO}_{3}\right)$ with characteristic fragment ions at $m / z 217.0189\left(\left[\mathrm{M}-\mathrm{H}-\mathrm{CO}_{2}\right]^{-}\right), 181.0569$ $\left(\left[\mathrm{M}-\mathrm{H}-\mathrm{SO}_{3}\right]^{-}\right), 137.0659\left(\left[\mathrm{M}-\mathrm{H}-\mathrm{SO}_{3}-\mathrm{CO}_{2}\right]^{-}\right)$and $123.0520\left(\left[\mathrm{M}-\mathrm{H}-\mathrm{SO}_{3}-\mathrm{CO}_{2}-\mathrm{CH}_{2}\right]^{-}\right)$. According to the previous report [44], M135 was tentatively identified as homovanillic acid sulphate.

Metabolites having the aglycone of dihydrocaffeic acid: M136-M138. M136 showed $[\mathrm{M}-\mathrm{H}]^{-}$ at $m / z 181.0504$ (predicted to be $\mathrm{C}_{9} \mathrm{H}_{9} \mathrm{O}_{4}$ ) and characteristic fragment ion at $m / z 137.0642$ ([M $-\mathrm{H}-$ $\left.\mathrm{CO}_{2}\right]^{-}$) was observed in the NI MS ${ }^{2}$ spectra. According to a previous report [45], we identified M136 as dihydrocaffeic acid. M137-M138 yielded [aglycone $-\mathrm{H}^{-}$at $\mathrm{m} / \mathrm{z} 181.05$ by neutral loss of $79.95 \mathrm{Da}$ and were tentatively identified as dihydrocaffeic acid sulphate.

Metabolites having the aglycone of caffeic acid: M139-M145. M139-M141 showed [M - H $]^{-}$at $m / z 238.07$ (predicted to be $\mathrm{C}_{11} \mathrm{H}_{12} \mathrm{NO}_{5}$ ) and yielded [aglycone $\left.-\mathrm{H}\right]^{-}$at $m / z 179.04$ in the MS ${ }^{2}$ spectra by neutral loss of $59.03 \mathrm{Da}\left(\mathrm{C}_{2} \mathrm{H}_{5} \mathrm{NO}\right)$. The aglycone had the same molecular formula and characteristic fragment ions as caffeic acid. Therefore, M139-M141 were designated caffeic acid acetyl amination 
metabolites. M142-M143 showed [M - H] $]^{-}$at $m / z$ 197.05, which is $18.01 \mathrm{Da}\left(\mathrm{H}_{2} \mathrm{O}\right)$ more than caffeic acid; thus, they were tentatively identified as hydration metabolites of caffeic acid. Based on the ClogP rule, M142 ( $\left.\mathrm{t}_{\mathrm{R}}=11.692\right)$ was 3-(3,4-dihydroxyphenyl)-3-hydroxypropanoic acid $(\mathrm{C} \log \mathrm{P}=-0.6414)$ and M143 ( $\left.t_{R}=12.658\right)$ was 3-(3,4-dihydroxyphenyl)-2-hydroxypropanoic acid $(\mathrm{ClogP}=-0.5798)$. M144-M145 showed [M - H] ${ }^{-}$at $m / z 277.00$, and the [aglycone $\left.-\mathrm{H}\right]^{-}$at $m / z 197.05$ was formed by the loss of 79.95 Da. Therefore, M144-M145 were tentatively identified as the sulphates of caffeic acid hydrate.

Metabolites having the aglycone of ferulic acid: M146-M148. The molecular formula of M146 was calculated to be $\mathrm{C}_{10} \mathrm{H}_{12} \mathrm{O}_{7} \mathrm{~S}$. The [aglycone $\left.-\mathrm{H}\right]^{-}$at $m / z 195.0681\left(\mathrm{C}_{10} \mathrm{H}_{11} \mathrm{O}_{4}\right)$ was formed by the loss of $\mathrm{SO}_{3}$ (79.95Da). Characteristic fragment ions at $m / z 195.0681,151.0845,149.0632,136.0607$ and 119.0578 were observed in NI MS² spectra. According to a previous report [45], we identified M146 as dihydrogen ferulic acid sulphate. M147-M148 showed [M - H] $]^{-}$at $m / z 291.02$ and the [aglycone $-\mathrm{H}]^{-}$at $m / z 211.06\left(\mathrm{C}_{10} \mathrm{H}_{11} \mathrm{O}_{5}\right)$ was formed by loss of $\mathrm{SO}_{3}(79.95 \mathrm{Da})$, which was $18.01 \mathrm{Da}$ $\left(\mathrm{H}_{2} \mathrm{O}\right)$ more than ferulic acid $\left(\mathrm{C}_{10} \mathrm{H}_{9} \mathrm{O}_{4}\right)$. Therefore, these metabolites were tentatively identified as the sulphates of ferulic acid hydrate.

In NI MS ${ }^{2}$ spectra of M149-M155, the same [aglycone $\left.-\mathrm{H}\right]^{-}(m / z$ 123.05) was observed with a molecular formula identical to hydroxybenzyl alcohol. We therefore deduced that they were conjugates of hydroxybenzyl alcohol [46]. According to characteristic neutral losses, M149 was tentatively identified as a sulphate of hydroxybenzyl alcohol, M150-M151 were identified as glucuronides of hydroxybenzyl alcohol and M152-M153 were identified as hydroxybenzyl alcohol glucuronide sulphates.

M154-M155 showed $[\mathrm{M}-\mathrm{H}]^{-}$at $m / z 217.02$ (predicted to be $\mathrm{C}_{8} \mathrm{H}_{9} \mathrm{O}_{5} \mathrm{~S}$ ), and yielded [aglycone $\left.-\mathrm{H}\right]^{-}$ at $m / z 137.07$ by neutral loss of $79.95 \mathrm{Da}$. The aglycone was $14.01 \mathrm{Da}\left(\mathrm{CH}_{2}\right)$ more than hydroxybenzyl alcohol, so the compounds were tentatively identified as sulphates of methyl hydroxybenzyl alcohol.

Metabolites having the aglycone of hydroxybenzoic acid: M156-M159. M156-M159 yielded [aglycone $-\mathrm{H}^{-}$by loss of $79.95 \mathrm{Da}$ and so were sulphate conjugates. From the [aglycone $\left.-\mathrm{H}\right]^{-}$ of M156-M157 at $m / z 137.03\left(\mathrm{C}_{7} \mathrm{H}_{6} \mathrm{O}_{3}\right)$, these were identified as 3/4-hydroxybenzoic acid sulphates according to a previous report [39]. From the [aglycone $-\mathrm{H}]^{-}$of $\mathbf{M 1 5 8 - M 1 5 9}$ at $m / z 167.04\left(\mathrm{C}_{8} \mathrm{H}_{8} \mathrm{O}_{4}\right)$, they could identify as vanillic acid sulphate and isovanillic acid sulphate according to the previous report [39].

\subsubsection{Identification of 32 Metabolites (M160-M191) Formed through Dimerization}

In total, 32 metabolites of dimerization (31 new compounds), including 10 taxifolin dimer derivatives and sulphates and 22 methyl taxifolin dimer derivatives and sulphates, were identified.

Dimers having the aglycone of taxifolin: M160-M169. The characteristic fragment ions of taxifolin at $m / z$ 303.05, $\mathrm{m} / \mathrm{z} 285.04$ and $\mathrm{m} / \mathrm{z} 241.05$ were observed in the NI MS ${ }^{2}$ spectra of M160-M169. We therefore deduced that their structures contained taxifolin, and that they were taxifolin dimer derivatives. The molecular formula of $\mathbf{M 1 6 0}$ was calculated to be $\mathrm{C}_{31} \mathrm{H}_{24} \mathrm{O}_{13}$ and, when compared with the molecular formula $\left(\mathrm{C}_{15} \mathrm{H}_{12} \mathrm{O}_{7}\right)$ of taxifolin, we predicted that $\mathbf{M 1 6 0}$ might be a dimer of taxiflolin and dehydroxylated methyl taxifolin. However, the site of dimerization was ambiguous. Only two forms of coupling bond are found between monomers of biflavonoids, namely C-C coupling and C-O coupling. In the NI MS ${ }^{2}$ spectra of M160, the relative abundance of $\mathrm{m} / \mathrm{z} 303.0557$ was less than $5 \%(4.08 \%)$, thus implying that the coupling bond between two monomers was extremely difficult to cleave [47]. Therefore, the dimer was considered to have formed through C-C coupling. One possible structure of M160 and its fragmentation pathways are shown in Figure S3. Similar to M160, we predicted that M161-M162 might be the dimers of taxiflolin and methyltaxifolin formed through C-C coupling. M163 might be a dimer of taxiflolin and dimethyltaxifolin formed through C-C coupling. M164-M166 were tentatively identified as sulphates of dimers of taxiflolin and methyltaxifolin. M167-M169 were tentatively identified as sulphate of dimers of taxiflolin and dimethyltaxifolin. 
Dimers having the aglycone of methyltaxifolin: M170-M191. The characteristic fragment ions of methyl taxifolin at $\mathrm{m} / \mathrm{z} 317.06, \mathrm{~m} / \mathrm{z} 299.05$ and $\mathrm{m} / \mathrm{z} 289.07$ were observed in the NI MS 2 spectra of M170-M191 (except M170, M172, M175). Similar to M160, we predicted that M170-M172 might be dimers of methyltaxiflolin and dehydroxylated methyltaxifolin. M171 was identified as a dimer formed through C-O coupling. M173-M176 might be dimers of methylquercetin and methyl-taxifolin. Among these, M174 was identified as a dimer formed through C-C coupling while M173, M175 and M176 were identified as dimers formed through C-O coupling. M177-M179 might be dimers of methyl taxiflolin and dehydroxylated dimethyltaxifolin formed through C-O coupling. M180 and M181 might be dimers of methyltaxiflolin and methyltaxifolin formed through $\mathrm{C}-\mathrm{O}$ and $\mathrm{C}-\mathrm{C}$ coupling, respectively. M182-M183 might be dimers of methyltaxiflolin and dimethylquercetin formed through C-O coupling. M184-M190 might be dimers of methyltaxiflolin and dimethyl- taxifolin; M190 was formed through C-C coupling while the other metabolites were formed through C-O coupling. M191 was tentatively identified as a sulphate of dimers of methyltaxiflolin and dehydroxylated methyltaxifolin.

In total, 32 dimers were newly identified as metabolites of taxifolin, and this is the first report of dimers formed as metabolites of flavanonol in vivo. To the best of our knowledge, the number of dimers found is the largest in metabolism studies to date, although six honokiol dimers were previously identified from the faeces of rats [48] and seven dimer metabolites of calycosin were identified in a rat hepatic $9000 \times g$ supernatant incubation system [47]. Dimers found in such large numbers may have important roles in pharmacological actions of taxifolin in vivo, because dimerization to homodimer or heterodimer (the twin drug approach) is a well known strategy in medicinal chemistry [49]. Therefore, the specific structure, formation mechanism and function of these metabolites require further study.

Unequivocal structure identification of the metabolites (known as the level 1 metabolite identification) is a fundamental issue in the field of drug metabolism research. Generally speaking, to solve this issue, the metabolites have to be prepared and purified from complex biological or chemical matrix, and then be analyzed by modern spectroscopic techniques such as NMR, circular dichroism (CD) and even X-ray diffraction. Unfortunately, the process is usually very difficult, because the contents of these metabolites in the biological matrix (such as urine, feces, plasma, etc.) are very low.

Since the substrate (original compound) is known in drug metabolism research, i.e., the exact chemical structure of the substrate is definite, the LC-HRMS ${ }^{\mathrm{n}}$ becomes the most common and effective method for quickly profiling and tentative identification of the metabolites to get a preliminary global view of the metabolic pathways of the original compound.

In this study, 191 metabolites of taxifolin were tentatively identified by their high resolution LC-MS $^{n}$ data. However, it's usually difficult or even impossible to determine regioisomers, stereoisomers and the exact metabolic site only by current MS techniques. Moreover, it is still a difficult problem to determine the exact sulphation site in flavonoids bearing a catechol moiety even by NMR technique. Fortunately, Purchartova et al. recently proposed a novel approach to solve this problem. They found that the methylation of flavonoid sulphates could be used for the direct and unequivocal determination of the position of sulphates in quercetin derivates by NMR [50]. This method is very useful for further determination of the specific structure of sulphates. According to their report, taxifolin can be metabolized to $4^{\prime}-O$-sulphate and $3^{\prime}-O$-sulphate in a ratio of 80:20 by bacterial aryl sulfotransferase from Desulfitobacterium hafniense. Besides, rat aryl sulfotransferase AstIV (EC 2.8.2.1) expressed recombinantly in Escherichia coli can biotransform taxifolin into taxifolin $3^{\prime}-O$-sulphate and quercetin $3^{\prime}-O$-sulphate [50]. These results imply that the metabolism of taxifolin is species-dependent. In addition, we also find that taxifolin can be metabolized to its sulphates (e.g., M3-M11) and quercetin sulphates (e.g., M71-M75), which is consistent with the results of rat AstIV, indicating the similarity between rat and recombinant rat AstIV.

There are four optical isomers of taxifolin because C-2 and C-3 are chiral centers, and we found two isomers metabolites (M1, M2) of taxifolin in this study. Since taxifolin has five hydroxyl groups, five sulphates could be formed at most. However, we have found nine taxifolin sulphates (M3-M11) based on LC-HRMS ${ }^{n}$ data, which indicates that the metabolites should include optical isomers. Because 
the amount of metabolites are small, we were not able to isolate sufficient metabolites and determine their exact structures. It needs more work and time to determine their exact structures by moder spectroscopic techniques in future.

\subsection{Distribution of the Metabolites of Taxifolin in Rats}

The distributions of 191 metabolites in eight rat organs (heart, liver, spleen, lung, kidney, brain, stomach and small intestine) were reported for the first time (shown in Table 3).

Table 3. Distribution of taxifolin and its 46 metabolites in rats.

\begin{tabular}{|c|c|c|c|c|c|c|c|c|}
\hline No. & Heart & Liver & Spleen & Lung & Kindey & Brain & Stomach & Intestine \\
\hline TAX & $\Delta$ & $\Delta$ & $\Delta$ & $\Delta$ & $\Delta$ & $\Delta$ & $\Delta$ & $\Delta$ \\
\hline M2 & - & $\Delta$ & - & $\Delta$ & $\Delta$ & $\Delta$ & $\Delta$ & $\Delta$ \\
\hline M5 & - & $\Delta$ & - & - & $\Delta$ & - & $\Delta$ & - \\
\hline M7 & - & - & - & - & - & - & - & $\Delta$ \\
\hline M11 & $\Delta$ & $\Delta$ & $\Delta$ & $\Delta$ & $\Delta$ & - & $\Delta$ & $\Delta$ \\
\hline M18 & $\Delta$ & $\Delta$ & $\Delta$ & $\Delta$ & $\Delta$ & - & $\Delta$ & $\Delta$ \\
\hline M19 & $\Delta$ & $\Delta$ & $\Delta$ & $\Delta$ & $\Delta$ & - & $\Delta$ & $\Delta$ \\
\hline M20 & - & - & $\Delta$ & $\Delta$ & $\Delta$ & - & $\Delta$ & $\Delta$ \\
\hline M21 & - & $\Delta$ & $\Delta$ & $\Delta$ & $\Delta$ & - & $\Delta$ & $\Delta$ \\
\hline M22 & $\Delta$ & - & - & - & - & - & - & - \\
\hline M23 & - & $\Delta$ & - & - & $\Delta$ & - & $\Delta$ & $\Delta$ \\
\hline M24 & - & $\Delta$ & - & - & - & - & - & $\Delta$ \\
\hline M25 & - & $\Delta$ & - & - & $\Delta$ & - & $\Delta$ & $\Delta$ \\
\hline M28 & - & - & - & - & $\bar{\Delta}$ & - & $\bar{\Delta}$ & - \\
\hline M29 & - & - & - & - & - & - & - & $\Delta$ \\
\hline M30 & - & - & - & - & $\Delta$ & - & $\Delta$ & $\Delta$ \\
\hline M31 & - & - & - & - & - & - & $\Delta$ & $\Delta$ \\
\hline M32 & - & $\Delta$ & - & - & $\Delta$ & - & - & $\Delta$ \\
\hline M33 & $\Delta$ & $\Delta$ & $\Delta$ & $\Delta$ & $\Delta$ & $\Delta$ & $\Delta$ & $\Delta$ \\
\hline M34 & - & $\Delta$ & $\Delta$ & $\Delta$ & $\Delta$ & $\Delta$ & $\Delta$ & $\Delta$ \\
\hline M35 & - & $\Delta$ & - & - & $\Delta$ & - & $\Delta$ & $\Delta$ \\
\hline M36 & - & - & - & - & - & - & $\Delta$ & - \\
\hline M42 & - & $\Delta$ & - & - & $\Delta$ & - & $\Delta$ & - \\
\hline M43 & $\Delta$ & - & $\Delta$ & $\Delta$ & - & - & - & $\Delta$ \\
\hline M44 & - & $\Delta$ & - & - & $\Delta$ & - & $\Delta$ & - \\
\hline M45 & - & $\Delta$ & - & - & $\Delta$ & - & $\Delta$ & $\Delta$ \\
\hline M48 & - & $\Delta$ & - & $\Delta$ & $\Delta$ & - & $\Delta$ & $\Delta$ \\
\hline M49 & $\Delta$ & $\Delta$ & $\Delta$ & $\Delta$ & $\Delta$ & - & $\Delta$ & $\Delta$ \\
\hline M50 & - & $\Delta$ & $\Delta$ & $\Delta$ & $\Delta$ & - & $\Delta$ & $\Delta$ \\
\hline M51 & - & - & - & - & - & - & - & $\Delta$ \\
\hline M52 & - & $\Delta$ & - & - & $\Delta$ & - & $\Delta$ & $\Delta$ \\
\hline M65 & - & - & - & - & - & - & $\Delta$ & $\Delta$ \\
\hline M70 & - & - & - & - & - & - & $\Delta$ & $\Delta$ \\
\hline M72 & - & - & - & - & $\Delta$ & - & - & $\Delta$ \\
\hline M75 & - & - & - & - & $\Delta$ & - & - & $\Delta$ \\
\hline M80 & - & - & - & - & $\Delta$ & - & - & $\Delta$ \\
\hline M84 & - & $\Delta$ & - & - & $\Delta$ & - & $\Delta$ & $\Delta$ \\
\hline M86 & - & - & - & - & $\Delta$ & - & - & - \\
\hline M87 & - & - & - & - & - & - & - & $\Delta$ \\
\hline M91 & - & - & - & - & - & - & - & $\Delta$ \\
\hline M105 & - & $\Delta$ & - & - & $\Delta$ & - & $\Delta$ & $\Delta$ \\
\hline M109 & - & - & - & - & $\Delta$ & - & - & $\Delta$ \\
\hline M118 & - & - & - & - & - & - & - & $\Delta$ \\
\hline M150 & - & - & - & - & $\Delta$ & - & - & - \\
\hline M151 & - & - & - & - & $\Delta$ & - & - & - \\
\hline M161 & - & - & - & - & - & - & $\Delta$ & - \\
\hline M162 & - & - & - & - & - & - & $\Delta$ & - \\
\hline SUM & 7 & 22 & 10 & 12 & 31 & 3 & 29 & 35 \\
\hline
\end{tabular}

In total 46 metabolites were detected in eight organs, and there were 35 metabolites in the small intestine, 31 in the kidneys, 29 in the stomach, 22 in the liver, 12 in the lungs, 10 in the spleen, seven in the heart, and three in the brain. Therefore, the small intestine, kidney, stomach and liver were the 
main organs for the distribution of the 46 metabolites of taxifolin. The methylated metabolite M33 was observed in all eight organs. M11, M18, M19, M34 and M49 were detected in seven organs. In total, 19 metabolites (M2, M11, M18-M21, M23, M25, M33-M35, M43, M45, M48-M50, M52, M84, M105) can be found in more than three organs. Therefore, these 19 metabolites were distributed more widely than the other metabolites, and they might contribute to the pharmacological activities of taxifolin in vivo.

\subsection{Bioactivities of the Metabolites of Taxifolin}

Among the metabolites of taxifolin, the nine phase I metabolites, taxifolin enantiomers (M1 and M2), quercetin (M70), eriodictyol (M104), dihydrokaempferol (M105), 3/4-hydroxyphenylpropionic acid (M122, M123), dihydroxyphenylacetic acid (M131) and dihydrocaffeic acid (M136), and the eight phase II metabolites, quercetin- $4^{\prime}-O$-sulphate (M73), quercetin- $3^{\prime}$-O-sulphate (M74), quercetin-3-O-sulphate (M75), quercetin glucuronide (M76), isorhamnetin (M80), isorhamnetin-3-O-sulphate (M83), isorhamnetin disulphate (M85) and luteolin-7-O-glucuronide (M113), have similar bioactivities to taxifolin according to the literature (Supplemental data Table S2). The activities of 17 bioactive metabolites can cover all biological activities (about 12 in total) of taxifolin, and the number of bioactive metabolites identified appears to be the largest reported in a metabolic study of a single compound. Hence, we considered that these active metabolites were the effective forms of taxifolin and could exert their in vivo effects simultaneously with taxifolin or successively.

\subsection{Prediction of Taxifolin Metabolite Targets}

Among the 191 metabolites, the specific structures of 63 were identified tentatively by their HRMS data, reference compounds, and previous studies (detailed in Table 1). The potential targets of taxifolin and 63 metabolites were predicted using the PharmMapper server. The predicted results showed that more than 60 metabolites have the same five targets: actin, alpha skeletal muscle (target 1), cystic fibrosis transmembrane conductance regulator (target 2), UDP-glucose 4-epimerase (target 3), nucleoside diphosphate kinase (target 4), and cytosolic and pancreatic ribonuclease (target 5). This finding indicates that these metabolites may act on the same target in vivo. According to the literature, some metabolites have the same target as taxifolin; these reported targets are summarised in Table S3. For example, taxifolin, M70 and M80 all target phosphoinositide 3-kinase (PI3K) to suppress cancer $[11,51,52]$.

Five of the top 300 PharmMapper-predicted target proteins of quercetin (M70) are reported in the literature: angiotensin-converting enzyme [53], glycogen synthase kinase-3 beta [54], beta-lactamase [55], beta-secretase 1 [56] and aspartate aminotransferase [57], as described in Table S4. Among these, glycogen synthase kinase- 3 beta is a well-established target related to cancer. A total of 41 metabolites were predicted to act via this target, and six metabolites were reported to exert antitumor activity. These results indicate the reliability of this server tool and indicate that these compounds may exert the same pharmacological effects on the same targets.

We also considered the structural similarity of the 63 identified metabolites. Their chemical structures have several common fragments, summarized as follows (and detailed in Table 4): four metabolites, M33, M34, M105 and M109, have fragment 1 (in red); six metabolites, M70, M73, M74, M80, M84 and M86, include fragment 2 (in red); eighteen metabolites, M33, M34, M36, M70, M73, M74, M75, M80, M83, M84, M86, M104, M105, M107, M109, M112, M114 and M115, contain fragment 3 (in red); and fourteen metabolites, M35, M36, M71, M72, M75, M80, M104, M106, M113, M131, M134, M136, M142 and M143, include fragment 4 (in red). Metabolites with the same fragment may contain the same pharmacological groups in their structures and act at the same targets with the same effects. For example, according to the literature, among the eighteen metabolites with fragment 3, eight metabolites (M70 [58], M73 [59], M74 [59], M75 [60], M80 [61], M83 [59], M104 [58] and M105 [62]) exhibit antioxidant activity and five metabolites (M70 [63], M75 [64], M80 [63], M104 [65] and M105 [66]) exhibit anti-inflammatory effects. Therefore, we speculated that other 
metabolites with the same fragment 3 may also exhibit the same bioactivities because they may act on the same individual targets.

Table 4. The common fragments (in red) and their related metabolites.

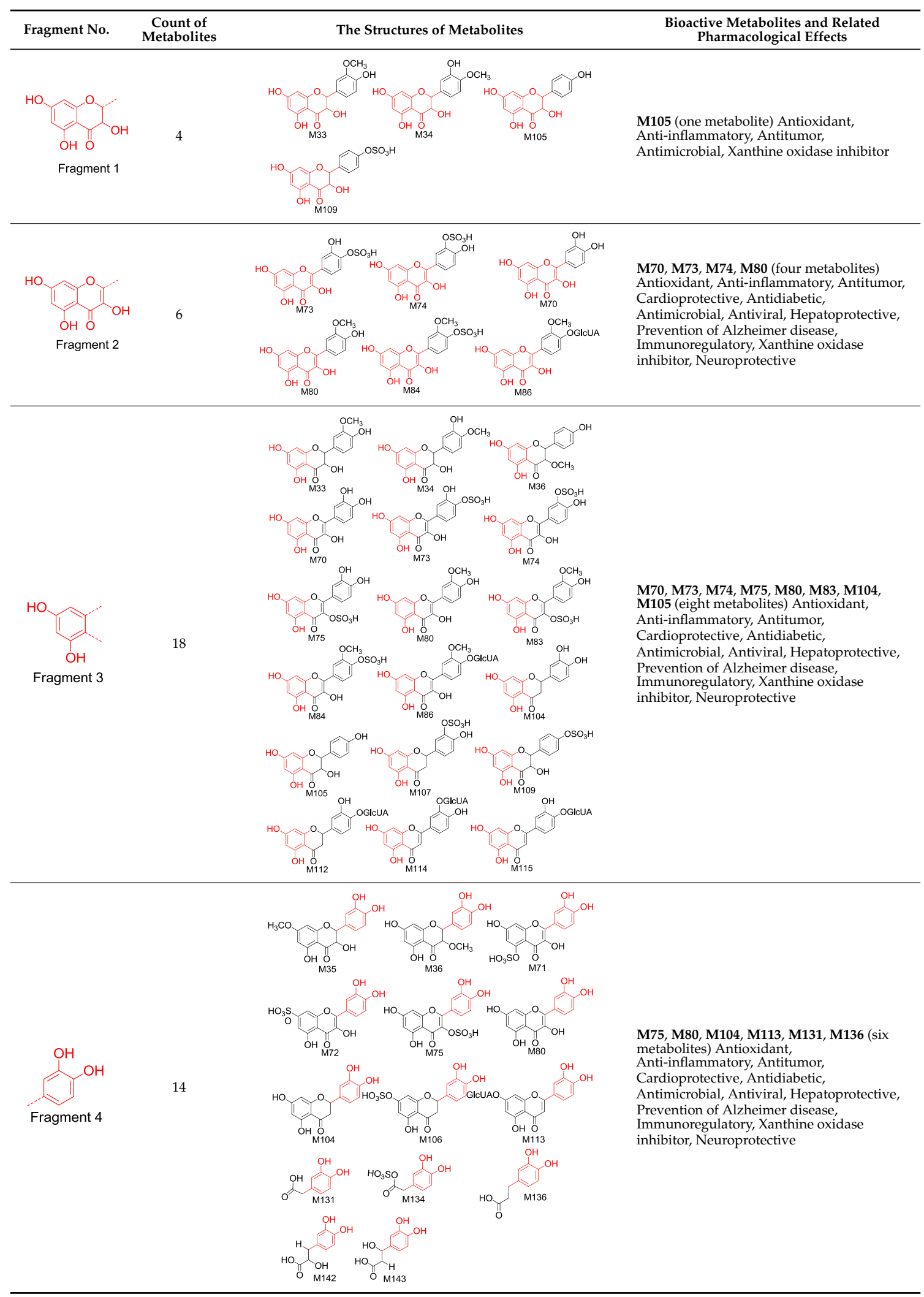




\section{Materials and Methods}

\subsection{Chemicals and Reagents}

(2R,3R)-(+)-Taxifolin (purity > 98\%) was purchased from Chengdu Must Bio-technology Co., Ltd (Chengdu, China) and used as the experiment source of taxifolin in the study. Quercetin and dihydrokaempferol were isolated in our laboratory, and the purities of these two standards were $>98 \%$ as determined by high-performance liquid chromatography coupled with diode array detector analysis (area normalization method). Formic acid (Roe Scientific Inc., Newark, NJ, USA), acetonitrile (Fisher Chemicals, Fairlawn, NJ, USA), and methanol (Tianjin Damao Chemicals, Tianjin, China) were of HPLC grade. Ultrapure water was prepared using a Milli-Q water purification system (Millipore, Billerica, MA, USA). Analytical-grade sodium carboxymethyl cellulose (CMC-Na) was purchased from Tianjin Guangfu Fine Chemical Research Institute (Tianjin, China). All other reagents and chemicals were of analytical grade.

\subsection{Animals and Drug Administration}

Twelve male Sprague-Dawley rats (weighing 180-220 g) were obtained from the Experimental Animal Center of Peking University Health Science Center (Beijing, China). The rats were maintained in metabolic cages (type DXL-DL, Suzhou Fengshi Laboratory Animal Equipment Co. Ltd, Suzhou, China) and acclimatized to the facilities for 5 days prior to experiments. All rats were housed in an environmentally controlled animal room, with food and water provided ad libitum. The rats were randomly divided into two groups (six rats per group), a drug group and a blank group. Taxifolin was suspended in 0.5\% CMC-Na solution and orally administered to the drug group at a dose of $200 \mathrm{mg} / \mathrm{kg}$ body weight, while blank group rats were orally administered $0.5 \%$ CMC-Na solution at the same volume. All rats were dosed once a day (at 9:00 a.m.) for 3 days. All animal treatments were conducted according to the Guide for the Care and Use of Laboratory Animals of the US National Institutes of Health. The animal research protocols were approved by the Biomedical Ethical Committee of Peking University (approval no. LA2015134).

\subsection{Urine and Faeces Samples Collection and Preparation}

During the administration period, urine and faeces samples from animals in the drug and blank groups were collected at 0-24 h after the first and second dosing, respectively. The urine samples were collected every $6 \mathrm{~h}$ from the urine collection tube (pre-filled with a small volume of methanol as preservative), a 1-fold volume of methanol was added, and samples were temporarily stored at $4{ }^{\circ} \mathrm{C}$. Finally, all urine samples from the same group were merged into one sample and immediately evaporated to dryness at $40^{\circ} \mathrm{C}$ under reduced pressure by a rotator evaporator. The dried sample was then extracted ultrasonically with a 4-fold volume of methanol for $30 \mathrm{~min}$ using an ultrasonic cleaner (at about $25^{\circ} \mathrm{C}$ ) and the extract was centrifuged at $5000 \mathrm{rpm}$ for $15 \mathrm{~min}$. Subsequently, the supernatant was dried in a vacuum at $40{ }^{\circ} \mathrm{C}$. Each $1 \mathrm{~g}$ residue was reconstituted in $2.0 \mathrm{~mL}$ methanol and filtered through a $0.45-\mu \mathrm{m}$ Millipore filter before undergoing LC-MS analysis.

Faecal samples were collected every $6 \mathrm{~h}$ and dried immediately using an electro-thermostatic blast oven at $40{ }^{\circ} \mathrm{C}$. Finally, all faecal samples from the same group were merged into one sample. The dry sample was ground to powder, and $3.0 \mathrm{~g}$ powder from each group was mixed with $15 \mathrm{~mL}$ of methanol and extracted ultrasonically for $30 \mathrm{~min}$ three times. Next, the extracts were centrifuged at $5000 \mathrm{rpm}$ for $15 \mathrm{~min}$ and the three supernatants were combined and evaporated to dryness under reduced pressure at $40{ }^{\circ} \mathrm{C}$. The resulting residue was dissolved in $3.0 \mathrm{~mL}$ methanol and filtered through a $0.45-\mu \mathrm{m}$ Millipore filter, and the filtrate was then subjected to LC-MS analysis.

\subsection{Blood Sample Collection and Preparation}

Blood samples were collected into heparinized tubes using a heart puncture technique under anaesthesia at $0.5,1$, and $1.5 \mathrm{~h}$ (two rats were sacrificed at each time point) after the last administration 
and were centrifuged at $5000 \mathrm{rpm}, 4^{\circ} \mathrm{C}$ for $10 \mathrm{~min}$ to obtain plasma. Plasma samples from the same time point within each group were combined into one sample and stored at $-80{ }^{\circ} \mathrm{C}$ until processing. Upon thawing, $24 \mathrm{~mL}$ methanol was added to $6 \mathrm{~mL}$ of plasma $(2 \mathrm{~mL}$ plasma from each of the three time points combined) in an ultrasonic bath for $30 \mathrm{~min}$ at about $25^{\circ} \mathrm{C}$ and samples were then centrifuged at $5000 \mathrm{rpm}$ for $15 \mathrm{~min}$ to remove precipitated protein. Next, the supernatant was concentrated to a small volume under reduced pressure at $40{ }^{\circ} \mathrm{C}$, transferred to a clean conical tube and dried under a gentle stream of nitrogen gas at ambient temperature. The residue was then reconstituted in $300 \mu \mathrm{L}$ of methanol and filtered through a $0.45-\mu \mathrm{m}$ Millipore filter before undergoing LC-MS analysis.

\subsection{Organ Sample Collection and Preparation}

After collection of blood samples and rapid removal of blood from organs via heart perfusion (until the liver became yellow in colour), the heart, liver, spleen, lungs, kidneys, brain, stomach and small intestine were rapidly removed and flushed with cold normal saline (with repeated washing three times to remove surface blood and other material), dried with filter paper, and weighed. All organ samples were stored at $-80^{\circ} \mathrm{C}$ until further processing. The same organ samples from each group were combined into one sample and processed using a homogenizer following suspension in a four-fold (volume/mass organ wet weight) volume of cold normal saline. Next, a $6 \mathrm{~mL}$ aliquot of homogenate from each organ sample was added to $48 \mathrm{~mL}$ of methanol, extracted ultrasonically for $30 \mathrm{~min}$ at about $25^{\circ} \mathrm{C}$, and centrifuged at $5000 \mathrm{rpm}$ for $15 \mathrm{~min}$ to remove the protein. The supernatant was evaporated to a small volume under reduced pressure at $40{ }^{\circ} \mathrm{C}$ and transferred into a clean tube. The supernatant was then dried under a gentle flow of nitrogen at ambient temperature, the residue was reconstituted in $1 \mathrm{~mL}$ methanol, and filtered through a $0.45-\mu \mathrm{m}$ Millipore filter, and the filtrate was subjected to LC-MS analysis.

\subsection{Instruments and Conditions for HPLC-ESI-IT-TOF-MS}

HPLC-ESI-IT-TOF-MS ${ }^{n}$ analysis was performed on a Shimadzu HPLC instrument (consisting of two LC-20AD pumps, a CTO-20A column oven, an SIL-20AC autosampler, an SPD-M20A PDA detector and a CBM-20A system controller) coupled to an IT-TOF mass spectrometer (Shimadzu, Kyoto, Japan) through an ESI interface. The chromatographic separations were carried out on an Agilent Zorbax SB-C18 column $(250 \mathrm{~mm} \times 4.6 \mathrm{~mm}, 5 \mu \mathrm{m})$ maintained at $30^{\circ} \mathrm{C}$ and protected using an Agilent Security Guard column ( $4.0 \mathrm{~mm} \times 3.0 \mathrm{~mm}, 5 \mu \mathrm{m}$; Agilent, Waldbronn, Germany). The mobile phase consisted of $0.1 \%$ formic acid in water (A) and acetonitrile (B). The gradient was as follows: $0 \mathrm{~min}$, 2\% B; $15 \mathrm{~min}, 10 \% \mathrm{~B} ; 30 \mathrm{~min}, 15 \% \mathrm{~B} ; 45 \mathrm{~min}, 22 \% \mathrm{~B} ; 60 \mathrm{~min}, 35 \% \mathrm{~B} ; 70 \mathrm{~min}, 55 \% \mathrm{~B} ; 85 \mathrm{~min}, 82 \% \mathrm{~B}$; $86 \mathrm{~min}, 100 \% \mathrm{~B} ; 95 \mathrm{~min}, 100 \% \mathrm{~B} ; 96 \mathrm{~min}, 2 \% \mathrm{~B}$; and $105 \mathrm{~min}, 2 \% \mathrm{~B}(v / v)$. The volume injected was $20 \mu \mathrm{L}$. High-resolution mass spectra were recorded using an IT-TOF mass spectrometer programmed to carry out a full scan over $m / z$ 100-1500 Da (MS) and $m / z$ 50-1000 Da (MS ${ }^{2}$ and $\mathrm{MS}^{3}$ ) in both positive ion (PI) and negative ion (NI) detection mode. The flow velocity was maintained at $1.0 \mathrm{~mL} / \mathrm{min}$ and was spilt at $0.2 \mathrm{~mL} / \mathrm{min}$ through a flow divider to flow into the mass spectrometer. A trifluoroacetic acid sodium solution $(2.5 \mathrm{mM})$ was used to calibrate the mass range of $50-1500 \mathrm{Da}$. The other operating parameters were set as follows: interface voltage was $(+), 4.5 \mathrm{kV} ;(-),-3.5 \mathrm{kV}$; nebulizing nitrogen gas flow was $1.5 \mathrm{~L} / \mathrm{min}$; detector voltage was $1.70 \mathrm{kV}$; relative collision-induced dissociation energy was 50\%; and heat block and curved desolvation line temperature was $200{ }^{\circ} \mathrm{C}$. All data were recorded and processed using LCMS solution version 3.60, Formula Predictor version 1.2 and Accurate Mass Calculator software (Shimadzu, Kyoto, Japan).

\subsection{Prediction of Taxifolin Metabolite Targets}

The potential targets of the metabolites of taxifolin were predicted using PharmMapper server (provided by the Shanghai Institute of Materia Medica, Chinese Academy of Sciences). PharmMapper is available at http:/ /59.78.96.61/pharmmapper. 


\subsection{Determination of the Level of Identification for All Metabolites}

The definition of metabolite identification level reported in the literature was generally adopted [67]. However, considering the difference between the research field of drug metabolism and metabolomics, we tentatively modify and define the identification levels (a little different from that in [67]) as follows:

Level 1: The metabolites are identified by comparison with reference compounds.

Level 2: The metabolites are identified by comparison with reference literature or can be found in the Scifinder database.

Level 3: New metabolites/compounds that could not be found in the SciFinder database.

\section{Conclusions}

A total of 191 metabolites (including 153 flavonoids and 38 phenols) of taxifolin were tentatively identified, 154 of whom were new metabolites of taxifolin. Furthermore, 69 metabolites were new compounds that were not found in the SciFinder database, including 12 taxifolin conjugates, 22 methyl taxifolin derivatives, four phenolic acid derivatives and 31 dimers. To our knowledge, this is the first report of a single compound biotransformed into more than 100 metabolites in vivo.

The major metabolic reactions of taxifolin in rats included ring-cleavage, sulphation, glucuronidation, methylation and dimerization. Furthermore, acetylamination and pyroglutamic acid conjugation were new metabolic reactions not described in any previous metabolism studies.

A total of 17 metabolites had similar bioactivites to taxifolin. The PharmMapper prediction showed that more than 60 metabolites had the same five targets. This suggested that the effective forms [68] of taxifolin are not only the parent form, but also the metabolites arising from it in vivo. And moreover, the effective metabolites are much larger in number than that of the imagination. These metabolites may exert the same pharmacological effects as taxifolin on the same targets. We therefore speculated that they might play the same role as the parent form through an additive effect [69]. These findings enhance the understanding of taxifolin metabolism and may provide further evidence of the beneficial effects of taxifolin and its derivatives in foods and other supplements. The study outcomes indicate that the metabolites and biotransformation of those bioactive constituents in foods and herbs require increased attention, especially to evaluate the biological activity of their metabolites. Our results may also provide a scientific support for our hypothesis of the traditional Chinese medicines (TCMs) efficacy theory [68], whereby TCMs exert their effects through the additive effects of numerous effective forms (including numerous original constituents and metabolites) on the same target, with synergistic effects based on the overall action of the additive effects on individual targets. Namely, numerous effective forms of incalculable constituents and their metabolites might participate in the process of pharmacodynamic action and could work together like an "army group". Our results may also provide an explanation to the question of how TCMs can exert pharmacological actions when the blood concentrations of their pharmacodynamic substances (constituents or their metabolites) are usually very low.

Supplementary Materials: Supplementary materials can be accessed at: http:/ /www.mdpi.com/1420-3049/21/ 9/1209/s1.

Acknowledgments: This study was financially supported by Beijing Natural Science Foundation (Grant No. 7162111). We are grateful to Jun Li for her routine management and careful maintenance of the LC MS-IT-TOF instrument.

Author Contributions: P.Y. performed the animal experiment, analyzed data, interpreted results of experiments and prepared the manuscript; F.X. established the analytical method, performed the LC-MS analysis and revised the manuscript; H.-F.L., Y.W. and F.-C.L. performed the animal experiment; M.-Y.S., G.-X.L. and X.W. reviewed the final manuscript; S.-Q.C. designed the whole research and reviewed the final manuscript.

Conflicts of Interest: The authors declare no conflict of interest. 


\section{References}

1. Vrhovsek, U.; Masuero, D.; Gasperotti, M.; Franceschi, P.; Caputi, L.; Viola, R.; Mattivi, F. A versatile targeted metabolomics method for the rapid quantification of multiple classes of phenolics in fruits and beverages. J. Agric. Food. Chem. 2012, 60, 8831-8840. [CrossRef] [PubMed]

2. Schauss, A.G.; Tselyico, S.S.; Kuznetsova, V.A.; Yegorova, I. Toxicological and genotoxicity assessment of a dihydroquercetin-rich dahurian larch tree (Larix gmelinii Rupr) extract (Lavitol). Int. J. Toxicol. 2015, 34, 162-181. [CrossRef] [PubMed]

3. Slimestad, R.; Fossen, T.; Vagen, I.M. Onions: a source of unique dietary flavonoids. J. Agric. Food. Chem. 2007, 55, 10067-10080. [CrossRef] [PubMed]

4. Wallace, S.N.; Carrier, D.J.; Clausen, E.C. Batch solvent extraction of flavanolignans from milk thistle (Silybum marianum L. Gaertner). Phytochem. Anal. 2005, 16, 7-16. [CrossRef] [PubMed]

5. Trebaticka, J.; Kopasova, S.; Hradecna, Z.; Cinovsky, K.; Skodacek, I.; Suba, J.; Muchova, J.; Zitnanova, I.; Waczulikova, I.; Rohdewald, P.; et al. Treatment of ADHD with French maritime pine bark extract, Pycnogenol. Eur. Child Adoles. Psy. 2006, 15, 329-335. [CrossRef] [PubMed]

6. Kiehlmann, E.; Li, E.P.M. Isomerization of dihydroquercetin. J. Nat. Prod. 1995, 58, 450-455. [CrossRef]

7. Vega-Villa, K.R.; Remsberg, C.M.; Ohgami, Y.; Yanez, J.A.; Takemoto, J.K.; Andrews, P.K.; Davies, N.M. Stereospecific high-performance liquid chromatography of taxifolin, applications in pharmacokinetics, and determination in tu fu ling (Rhizoma Smilacis glabrae) and apple (Malus x domestica). Biomed. Chromatogr. 2009, 23, 638-646. [CrossRef] [PubMed]

8. Weidmann, A.E. Dihydroquercetin: More than just an impurity? Eur. J. Pharmacol. 2012, 684, $19-26$. [CrossRef] [PubMed]

9. Topal, F.; Nar, M.; Gocer, H.; Kalin, P.; Kocyigit, U.M.; Gulcin, I.; Alwasel, S.H. Antioxidant activity of taxifolin: an activity-structure relationship. J. Enzyme Inhib. Med. Chem. 2016, 31, 674-683. [CrossRef] [PubMed]

10. Willfoer, S.M.; Ahotupa, M.O.; Hemming, J.E.; Reunanen, M.H.T.; Eklund, P.C.; Sjoeholm, R.E.; Eckerman, C.S.E.; Pohjamo, S.P.; Holmbom, B.R. Antioxidant activity of knotwood extractives and phenolic compounds of selected tree species. J. Agric. Food. Chem. 2003, 51, 7600-7606. [CrossRef] [PubMed]

11. Oi, N.; Chen, H.; Kim, M.O.; Lubet, R.A.; Bode, A.M.; Dong, Z. Taxifolin suppresses UV-induced skin carcinogenesis by targeting EGFR and PI3K. Cancer Prev. Res. 2012, 5, 1103-1114. [CrossRef] [PubMed]

12. Delporte, C.; Backhouse, N.; Erazo, S.; Negrete, R.; Vidal, P.; Silva, X.; Lopez-Perez, J.L.; Feliciano, A.S.; Munoz, O. Analgesic-antiinflammatory properties of Proustia pyrifolia. J. Ethnopharmacol. 2005, 99, 119-124. [CrossRef] [PubMed]

13. Gocer, H.; Topal, F.; Topal, M.; Kucuk, M.; Teke, D.; Gulcin, I.; Alwasel, S.H.; Supuran, C.T. Acetylcholinesterase and carbonic anhydrase isoenzymes I and II inhibition profiles of taxifolin. J. Enzyme Inhib. Med. Chem. 2015, 1-7. [CrossRef] [PubMed]

14. Fedosova, N.F.; Alisievich, S.V.; Lyadov, K.V.; Romanova, E.P.; Rud'Ko, I.A.; Kubatiev, A.A. Mechanisms underlying diquertin-mediated regulation of neutrophil function in patients with non-insulin-dependent diabetes mellitus. Bull. Exp. Biol. Med. 2004, 137, 143-146. [CrossRef] [PubMed]

15. Sun, X.; Chen, R.C.; Yang, Z.H.; Sun, G.B.; Wang, M.; Ma, X.J.; Yang, L.J.; Sun, X.B. Taxifolin prevents diabetic cardiomyopathy in vivo and in vitro by inhibition of oxidative stress and cell apoptosis. Food Chem. Toxicol. 2014, 63, 221-232. [CrossRef] [PubMed]

16. Ezzikouri, S.; Nishimura, T.; Kohara, M.; Benjelloun, S.; Kino, Y.; Inoue, K.; Matsumori, A.; Tsukiyama-Kohara, K. Inhibitory effects of pycnogenol on hepatitis C virus replication. Antiviral Res. 2015, 113, 93-102. [CrossRef] [PubMed]

17. Fongang, Y.S.F.; Bankeu, J.J.K.; Ali, M.S.; Awantu, A.F.; Zeeshan, A.; Assob, C.N.; Mehreen, L.; Lenta, B.N.; Ngouela, S.A.; Tsamo, E. Flavonoids and other bioactive constituents from Ficus thonningii Blume (Moraceae). Phytochem. Lett. 2015, 11, 139-145. [CrossRef]

18. Zhao, M.; Chen, J.; Zhu, P.; Fujino, M.; Takahara, T.; Toyama, S.; Tomita, A.; Zhao, L.; Yang, Z.; Hei, M.; et al. Dihydroquercetin (DHQ) ameliorated concanavalin A-induced mouse experimental fulminant hepatitis and enhanced HO-1 expression through MAPK/Nrf2 antioxidant pathway in RAW cells. Int. Immunopharmacol. 2015, 28, 938-944. [CrossRef] [PubMed] 
19. Guo, H.; Zhang, X.; Cui, Y.; Zhou, H.; Xu, D.; Shan, T.; Zhang, F.; Guo, Y.; Chen, Y.; Wu, D. Taxifolin protects against cardiac hypertrophy and fibrosis during biomechanical stress of pressure overload. Toxicol. Appl. Pharmacol. 2015, 287, 168-177. [CrossRef] [PubMed]

20. Dok-Go, H.; Lee, K.H.; Kim, H.J.; Lee, E.H.; Lee, J.; Song, Y.S.; Lee, Y.H.; Jin, C.; Lee, Y.S.; Cho, J. Neuroprotective effects of antioxidative flavonoids, quercetin, (+)-dihydroquercetin and quercetin 3-methyl ether, isolated from Opuntia ficus-indica var. saboten. Brain Res. 2003, 965, 130-136. [CrossRef]

21. Ahn, J.Y.; Choi, S.E.; Jeung, M.S.; Park, K.H.; Moon, N.J.; Joo, S.S.; Lee, C.S.; Choi, Y.W.; Li, K.; Lee, M.K.; et al. Effect of taxifolin glycoside on atopic dermatitis-like skin lesions in NC/Nga mice. Phytother. Res. 2010, 24, 1071-1077. [CrossRef] [PubMed]

22. Liang, L.; Gao, C.; Luo, M.; Wang, W.; Zhao, C.; Zu, Y.; Efferth, T.; Fu, Y. Dihydroquercetin (DHQ) induced HO-1 and NQO1 expression against oxidative stress through the Nrf2-dependent antioxidant pathway. J. Agric. Food Chem. 2013, 61, 2755-2761. [CrossRef] [PubMed]

23. Booth, A.N.; DeEds, F. The toxicity and metabolism of dihydroquercetin. J. Am. Pharm. Assoc. Am. Pharm. Assoc. 1958, 47, 183-184. [CrossRef] [PubMed]

24. Spencer, J.P.; Abd-el-Mohsen, M.M.; Rice-Evans, C. Cellular uptake and metabolism of flavonoids and their metabolites: Implications for their bioactivity. Arch. Biochem. Biophys. 2004, 423, 148-161. [CrossRef] [PubMed]

25. Yamamoto, M.; Jokura, H.; Hashizume, K.; Ominami, H.; Shibuya, Y.; Suzuki, A.; Hase, T.; Shimotoyodome, A. Hesperidin metabolite hesperetin-7-O-glucuronide, but not hesperetin-3'-O-glucuronide, exerts hypotensive, vasodilatory, and anti-inflammatory activities. Food Funct. 2013, 4, 1346-1351. [CrossRef] [PubMed]

26. Lotito, S.B.; Zhang, W.-J.; Yang, C.-S.; Crozier, A.; Frei, B. Metabolic conversion of dietary flavonoids alters their anti-inflammatory and antioxidant properties. Free Radic. Biol. Med. 2011, 51, 454-463. [CrossRef] [PubMed]

27. Wiczkowski, W.; Szawara-Nowak, D.; Topolska, J.; Olejarz, K.; Zieliński, H.; Pisku A, M.K. Metabolites of dietary quercetin: Profile, isolation, identification, and antioxidant capacity. J. Funct. Foods 2014, 11, 121-129. [CrossRef]

28. Tang, H.; Tang, L.; Xu, R.; Yang, J. Phase II metabolites of several flavonoids and their pharmacological activities. Zhongguo Xin Yao Za Zhi 2012, 21, 144-150.

29. Kawabata, K.; Mukai, R.; Ishisaka, A. Quercetin and related polyphenols: new insights and implications for their bioactivity and bioavailability. Food Funct. 2015, 6, 1399-1417. [CrossRef] [PubMed]

30. Wang, X.D. Studies on the Transport of Taxifolin and Astilbin in Vitro and Pharmacokinetics in Rats. Ph.D. Thesis, Zhejiang University, Zhejiang, China, 2009.

31. Duweler, K.G.; Rohdewald, P. Urinary metabolites of French maritime pine bark extract in humans. Pharmazie 2000, 55, 364-368. [PubMed]

32. Liang, J.; Xu, F.; Zhang, Y.Z.; Zang, X.Y.; Wang, D.; Shang, M.Y.; Wang, X.; Chui, D.H.; Cai, S.Q. The profiling and identification of the metabolites of (+)-catechin and study on their distribution in rats by

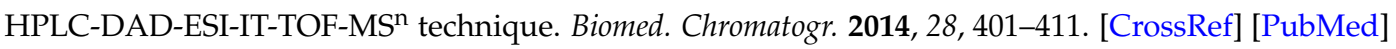

33. Yang, X.R.; Xu, F.; Li, D.P.; Lu, F.L.; Liu, G.X.; Wang, L.; Shang, M.Y.; Huang, Y.L.; Cai, S.Q. Metabolites of Siamenoside I and their distributions in rats. Molecules 2016, 21, 176. [CrossRef] [PubMed]

34. Blaut, M.; Schoefer, L.; Braune, A. Transformation of flavonoids by intestinal microorganisms. Int. J. Vitam. Nutr. Res. 2003, 73, 79-87. [CrossRef] [PubMed]

35. Vacek, J.; Papouskova, B.; Kosina, P.; Vrba, J.; Kren, V.; Ulrichova, J. Biotransformation of flavonols and taxifolin in hepatocyte in vitro systems as determined by liquid chromatography with various stationary phases and electrospray ionization-quadrupole time-of-flight mass spectrometry. J. Chromatogr. B 2012, 899, 109-115. [CrossRef] [PubMed]

36. Wang, X.D.; Meng, M.X.; Gao, L.B.; Liu, T.; Xu, Q.; Zeng, S. Permeation of astilbin and taxifolin in Caco-2 cell and their effects on the P-gp. Int. J. Pharm. 2009, 378, 1-8. [CrossRef] [PubMed]

37. Wang, X.D.; Xia, H.J.; Xing, F.; Deng, G.F.; Shen, Q.; Zeng, S. A highly sensitive and robust UPLC-MS with electrospray ionization method for quantitation of taxifolin in rat plasma. J. Chromatogr. B 2009, 877, 1778-1786. [CrossRef] [PubMed]

38. Pozharitskaya, O.N.; Karlina, M.V.; Shikov, A.N.; Kosman, V.M.; Makarova, M.N.; Makarov, V.G. Determination and pharmacokinetic study of taxifolin in rabbit plasma by high-performance liquid chromatography. Phytomedicine 2009, 16, 244-251. [CrossRef] [PubMed] 
39. Liang, J.; Xu, F.; Zhang, Y.Z.; Huang, S.; Zang, X.Y.; Zhao, X.; Zhang, L.; Shang, M.Y.; Yang, D.H.; Wang, X.; et al. The profiling and identification of the absorbed constituents and metabolites of paeoniae radix rubra decoction in rat plasma and urine by the HPLC-DAD-ESI-IT-TOF-MS ${ }^{n}$ technique: a novel strategy for the systematic screening and identification of absorbed constituents and metabolites from traditional Chinese medicines. J. Pharm. Biomed. Anal. 2013, 83, 108-121. [PubMed]

40. Ma, L.M.; Xu, F.; Li, F.C.; Wang, J.Z.; Shang, M.Y.; Liu, G.X.; Cai, S.Q. The profiling and identification of the metabolites of 8-prenylkaempferol and a study on their distribution in rats by high-performance liquid chromatography with diode array detection combined with electrospray ionization ion trap time-of-flight multistage mass spectrometry. Biomed. Chromatogr. 2015, 30, 175-190. [PubMed]

41. Van der Woude, H.; Boersma, M.G.; Vervoort, J.; Rietjens, I.M. Identification of 14 quercetin phase II mono- and mixed conjugates and their formation by rat and human phase II in vitro model systems. Chem. Res. Toxicol. 2004, 17, 1520-1530. [CrossRef] [PubMed]

42. Marmet, C.; Actis-Goretta, L.; Renouf, M.; Giuffrida, F. Quantification of phenolic acids and their methylates, glucuronides, sulfates and lactones metabolites in human plasma by LC-MS/MS after oral ingestion of soluble coffee. J. Pharm. Biomed. Anal. 2014, 88, 617-625. [CrossRef] [PubMed]

43. Uutela, P.; Reinila, R.; Harju, K.; Piepponen, P.; Ketola, R.A.; Kostiainen, R. Analysis of intact glucuronides and sulfates of serotonin, dopamine, and their phase I metabolites in rat brain microdialyzates by liquid chromatography-tandem mass spectrometry. Anal. Chem. 2009, 81, 8417-8425. [CrossRef] [PubMed]

44. Geng, J.L.; Dai, Y.; Yao, Z.H.; Qin, Z.F.; Wang, X.L.; Qin, L.; Yao, X.S. Metabolites profile of Xian-Ling-Gu-Bao capsule, a traditional Chinese medicine prescription, in rats by ultra performance liquid chromatography coupled with quadrupole time-of-flight tandem mass spectrometry analysis. J. Pharm. Biomed. Anal. 2014, 96, 90-103. [CrossRef] [PubMed]

45. Redeuil, K.; Smarrito-Menozzi, C.; Guy, P.; Rezzi, S.; Dionisi, F.; Williamson, G.; Nagy, K.; Renouf, M. Identification of novel circulating coffee metabolites in human plasma by liquid chromatography-mass spectrometry. J. Chromatogr. A 2011, 1218, 4678-4688. [CrossRef] [PubMed]

46. Schmidt, J.; Kotnik, P.; Trontelj, J.; Knez, Ž.; Mašič, L.P. Bioactivation of bisphenol A and its analogs (BPF, BPAF, BPZ and DMBPA) in human liver microsomes. Toxicol. In Vitro 2013, 27, 1267-1276. [CrossRef] [PubMed]

47. Zhang, Y.Z.; Xu, F.; Dong, J.; Liang, J.; Hashi, Y.; Shang, M.Y.; Yang, D.H.; Wang, X.; Cai, S.Q. Profiling and identification of the metabolites of calycosin in rat hepatic $9000 \mathrm{x} g$ supernatant incubation system and the metabolites of calycosin-7-O- $\beta$-D-glucoside in rat urine by HPLC-DAD-ESI-IT-TOF-MS ${ }^{n}$ technique. J. Pharm. Biomed. Anal. 2012, 70, 425-439. [CrossRef] [PubMed]

48. Dong, Y.; Tang, M.; Song, H.; Li, R.; Wang, C.; Ye, H.; Qiu, N.; Zhang, Y.; Chen, L.; Wei, Y. Characterization of metabolic profile of honokiol in rat feces using liquid chromatography coupled with quadrupole time-of-flight tandem mass spectrometry and ${ }^{13} \mathrm{C}$ stable isotope labeling. J. Chromatogr. B 2014, 953-954, 20-29. [CrossRef] [PubMed]

49. Contreras, J.; Sippl, W. Homo and heterodimer ligands: the twin drug approach. In The Practice of Medicinal Chemistry, 3rd ed.; Wermuth, C.G., Ed.; Elsevier Ltd.: London, UK, 2008; pp. 380-383.

50. Purchartova, K.; Valentova, K.; Pelantova, H.; Marhol, P.; Cvacka, J.; Havlicek, L.; Krenkova, A.; Vavrikova, E.; Biedermann, D.; Chambers, C.S.; et al. Prokaryotic and Eukaryotic Aryl Sulfotransferases: Sulfation of Quercetin and Its Derivatives. Chemcatchem 2015, 7, 3152-3162. [CrossRef]

51. Yoshizumi, M.; Tsuchiya, K.; Kirima, K.; Kyaw, M.; Suzaki, Y.; Tamaki, T. Quercetin inhibits Shc- and phosphatidylinositol 3-kinase-mediated c-Jun N-terminal kinase activation by angiotensin II in cultured rat aortic smooth muscle cells. Mol. Pharmacol. 2001, 60, 656-665. [PubMed]

52. Kim, J.E.; Lee, D.E.; Lee, K.W.; Son, J.E.; Seo, S.K.; Li, J.; Jung, S.K.; Heo, Y.S.; Mottamal, M.; Bode, A.M. Isorhamnetin suppresses skin cancer through direct inhibition of MEK1 and PI3-K. Cancer Prev. Res. 2011, 4, 582-591. [CrossRef] [PubMed]

53. Nicolau, M.; Dovichi, S.S.; Cuttle, G. Pro-inflammatory effect of quercetin by dual blockade of angiotensin converting-enzyme and neutral endopeptidase in vivo. Nutr. Neurosci. 2003, 6, 309-316. [CrossRef] [PubMed]

54. Johnson, J.L.; Rupasinghe, S.G.; Felicia, S.; Schuler, M.A.; Elvira, G.D.M. Citrus flavonoids luteolin, apigenin, and quercetin inhibit glycogen synthase kinase- $3 \beta$ enzymatic activity by lowering the interaction energy within the binding cavity. J. Med. Food 2011, 14, 325-333. [CrossRef] [PubMed] 
55. Boussoualim, N.; Abderrahmane, B. Kinetic study of different flavonoids as inhibitors of beta-lactamase enzyme. Afr. J. Biochem. Res. 2011, 321-327.

56. Song, K.S.; Choi, S.H.; Hur, J.M.; Park, H.J.; Yang, E.J.; Mook-Jung, I.; Yi, J.H.; Jun, M. Inhibitory effects of flavonoids isolated from leaves of Petasites japonicus on $\beta$-secretase (BACE1). Food Sci. Biotechnol. 2008, 17, 1165-1170.

57. Wan, Y.; Tang, M.H.; Chen, X.C.; Chen, L.J.; Wei, Y.Q.; Wang, Y.S. Inhibitory effect of liposomal quercetin on acute hepatitis and hepatic fibrosis induced by concanavalin A. Braz. J. Med. Biol. Res. 2014, 47, 655-661. [CrossRef] [PubMed]

58. Sawai, Y. NMR analytical approach to clarify the molecular mechanisms of the antioxidative and radical-scavenging activities of antioxidants in tea: Reaction of polyphenols with stable free radicals. Yasai Chagyo Kenkyusho Kenkyu Hokoku 2007, 6, 23-58.

59. Duenas, M.; Surco-Laos, F.; Gonzalez-Manzano, S.; Gonzalez-Paramas, A.M.; Santos-Buelga, C. Antioxidant properties of major metabolites of quercetin. Eur. Food Res. Technol. 2011, 232, 103-111. [CrossRef]

60. Yagi, A.; Uemura, T.; Okamura, N.; Haraguchi, H.; Imoto, T.; Hashimoto, K. Antioxidative sulfated flavonoids in leaves of Polygonum hydropiper. Phytochemistry 1994, 35, 885-887. [CrossRef]

61. Seo, K.; Yang, J.H.; Kim, S.C.; Ku, S.K.; Ki, S.H.; Shin, S.M. The antioxidant effects of isorhamnetin contribute to inhibit COX-2 expression in response to inflammation: a potential role of HO-1. Inflammation 2014, 37, 712-722. [CrossRef] [PubMed]

62. Lee, I.K.; Song, K.S.; Kim, C.J.; Kim, H.M.; Oh, G.T.; Yoo, I.D. Tumor cell growth inhibition and antioxidant activity of flavonoids from the stem bark of Cudrania tricuspidata. Han'guk Nonghwa Hakhoechi 1994, 37, 105-109.

63. Boesch-Saadatmandi, C.; Loboda, A.; Wagner, A.E.; Stachurska, A.; Jozkowicz, A.; Dulak, J.; Doring, F.; Wolffram, S.; Rimbach, G. Effect of quercetin and its metabolites isorhamnetin and quercetin-3-glucuronide on inflammatory gene expression: role of miR-155. J. Nutr. Biochem. 2011, 22, 293-299. [CrossRef] [PubMed]

64. Ksouri, R.; Ksouri, W.M.; Jallali, I.; Debez, A.; Magne, C.; Hiroko, I.; Abdelly, C. Medicinal halophytes: potent source of health promoting biomolecules with medical, nutraceutical and food applications. Crit. Rev. Biotechnol. 2012, 32, 289-326. [CrossRef] [PubMed]

65. Armah, F.A.; Annan, K.; Mensah, A.Y.; Amponsah, I.K.; Tocher, D.A.; Habtemariam, S. Erythroivorensin: A novel anti-inflammatory diterpene from the root-bark of Erythrophleum ivorense (A Chev.). Fitoterapia 2015, 105, 37-42. [CrossRef] [PubMed]

66. Tan, Y.; Liu, C.; Chen, R. Phenolic constituents from stem bark of Morus wittiorum and their anti-inflammation and cytotoxicity. Zhongguo Zhong Yao Za Zhi 2010, 35, 2700-2703. [PubMed]

67. Sumner, L.W.; Amberg, A.; Barrett, D.; Beale, M.H.; Beger, R.; Daykin, C.A.; Fan, T.W.M.; Fiehn, O.; Goodacre, R.; Griffin, J.L.; et al. Proposed minimum reporting standards for chemical analysis. Metabolomics 2007, 3, 211-221. [CrossRef] [PubMed]

68. Cai, S.Q.; Wang, X.; Shang, M.Y.; Xu, F.; Liu, G.X. "Efficacy theory" may help to explain characteristic advantages of traditional Chinese medicines. Zhongguo Zhong Yao Za Zhi 2015, 40, 3435-3443. [PubMed]

69. Li, C.; Xu, F.; Xie, D.M.; Jing, Y.; Shang, M.Y.; Liu, G.X.; Wang, X.; Cai, S.Q. Identification of absorbed constituents in the rabbit plasma and cerebrospinal fluid after intranasal administration of Asari Radix et Rhizoma by HS-SPME-GC-MS and HPLC-APCI-IT-TOF-MS ${ }^{\mathrm{n}}$. Molecules 2014, 19, 4857-4879. [CrossRef] [PubMed]

Sample Availability: Samples of the compounds are not available from the authors. 